$\underline{\underline{\text { IIIIIIII }}}$ Open Access Articles

\title{
Synthetic Variation and Structural Trends in Layered Two- Dimensional Alkylammonium Lead Halide Perovskites
}

The MIT Faculty has made this article openly available. Please share how this access benefits you. Your story matters.

\begin{tabular}{|l|l|}
\hline As Published & $10.1021 /$ acs.chemmater.9b01318 \\
\hline Publisher & American Chemical Society (ACS) \\
\hline \hline Version & Original manuscript \\
\hline Citable link & https://hdl.handle.net/1721.1/134629 \\
\hline Terms of Use & $\begin{array}{l}\text { Article is made available in accordance with the publisher's } \\
\text { policy and may be subject to US copyright law. Please refer to the } \\
\text { publisher's site for terms of use. }\end{array}$ \\
\hline
\end{tabular}




\title{
Synthetic Variation and Structural Trends in Layered Two- Dimensional Alkylammonium Lead Halide Perovskites
}

\author{
Watcharaphol Paritmongkol ${ }^{1,2}$, Nabeel S. Dahod ${ }^{2}$, Nannan $\mathrm{Mao}^{2,3}$, \\ Shao-Liang Zheng ${ }^{4}$, William A. Tisdale ${ }^{2 *}$ \\ ${ }^{1}$ Department of Chemistry, Massachusetts Institute of Technology, Cambridge, Massachusetts \\ 02139, United States \\ ${ }^{2}$ Department of Chemical Engineering, Massachusetts Institute of Technology, Cambridge, \\ Massachusetts 02139, United States \\ ${ }^{3}$ Department of Electrical Engineering and Computer Science, Massachusetts Institute of \\ Technology, Cambridge, Massachusetts 02139, United States \\ ${ }^{4}$ Department of Chemistry and Chemical Biology, Harvard University, Cambridge, Massachusetts \\ 02138, United States \\ *Correspondence to: tisdale@mit.edu
}

\begin{abstract}
We report the cooling-induced crystallization of layered two-dimensional lead halide perovskites with controllable inorganic quantum-well thickness $(n=1,2,3,4)$, organic spacer chain length (butyl-, pentyl-, hexylammonium), A-site cation (methylammonium, formamidinium), and halide anion (iodide, bromide). We report crystal structures for the iodide family as a function of these compositional parameters, and across their temperature dependent phase transitions. In general, lower symmetry crystal structures, increasing extents of organic-spacer interdigitation, and increasing organic-spacer corrugation tilts are observed at low temperature. In addition, greater structural distortions are seen in lead halide octahedra closest to the organic spacer layer, and larger- $n$ structures exhibit periodic variation in $\mathrm{Pb}$-I bond lengths. We also provide detailed guidance regarding the combination of synthetic parameters needed to achieve phase-pure crystals of each composition, and discuss difficulties encountered when trying to synthesize particular members of the $2 \mathrm{D}$ perovskite family containing formamidinium or cesium as the A-site cation. These results provide a foundation for understanding structural trends in 2D lead halide perovskites and the effect these trends have on their thermal, electrical, and optical properties.
\end{abstract}




\section{Introduction}

Layered two-dimensional (2D) lead halide perovskites (LHPs) have emerged as an alternative to their bulk or three-dimensional (3D) counterparts due to improved stability, ${ }^{1,2}$ rich structural flexibility, ${ }^{3,4}$ and greater tunability of optical properties. ${ }^{3,5}$ Their structures consist of $2 \mathrm{D}$ inorganic slabs of $\mathrm{ABX}_{3}$ perovskite unit cells separated by large monovalent cations. Their general formula is $\mathrm{L}_{2}\left[\mathrm{ABX}_{3}\right]_{\mathrm{n}-1} \mathrm{BX}_{4}$ or $\mathrm{L}_{2} \mathrm{~A}_{\mathrm{n}-1} \mathrm{~B}_{\mathrm{n}} \mathrm{X}_{3 \mathrm{n}+1}$, where $\mathrm{L}$ is a large monovalent cation, $\mathrm{A}$ is a small cation (i.e. methylammonium $\{\mathrm{MA}\}$, formamidinium $\{\mathrm{FA}\}$ or cesium $\{\mathrm{Cs}\}$ ), $\mathrm{B}$ is a divalent cation of lead $(\mathrm{Pb}), \mathrm{X}$ is a halide anion $(\mathrm{Cl}, \mathrm{Br}$, or $\mathrm{I})$, and $n$ determines the inorganic quantum-well thickness defined by the number of corner-sharing $\mathrm{BX}_{6}$ octahedral layers. Since $\mathrm{L}$ is usually a large organic molecule with an amine functional group in its protonated form, L acts as an electronically insulating organic spacer and 2D LHPs form natural quantum-well structures. Due to the spatial confinement and the low dielectric screening of organic spacers, 2D LHPs have high exciton binding energies ( $>400 \mathrm{meV}$ for single-layered 2D LHPs). ${ }^{6,7}$ Moreover, both optical and electronic properties of 2D LHPs can be tuned by varying the identities of each constituent ion (L, A, B, and $\mathrm{X}$ ) as well as $n \cdot{ }^{3,5}$ Hence, 2D LHPs have been applied in various optoelectronic devices, such as solar cells, ${ }^{1,2,8-12}$ light-emitting diodes, ${ }^{13-16}$ and photodetectors. ${ }^{17-20}$

Electrical and optical properties of 2D LHPs are determined by their structure. ${ }^{3,21-24}$ Structural evolution of 3D LHPs and single-layered $(n=1)$ 2D LHPs with the absence of small A-site cations has been extensively investigated and shows interesting temperature-dependent transformations. ${ }^{25-30}$ For example, 3D $\mathrm{MAPbI}_{3}$ perovskites adopt cubic, tetragonal, and orthorhombic phases at high, intermediate, and low temperatures, respectively ${ }^{25-27}$ On the other hand, the number of crystal phases and phase transition temperatures of single-layered $\left(\mathrm{C}_{\mathrm{m}} \mathrm{H}_{2 \mathrm{~m}+1} \mathrm{NH}_{3}\right)_{2} \mathrm{PbI}_{4}(m=4,5,6,7,8,9,10,12,14,16$, and 18) are dependent on the chain length 
of the organic spacer. ${ }^{28-30}$ Higher members $(n \geq 2)$ of 2 D LHPs contain both organic spacers and A-site cations, and lie in between these two limits. Interesting questions include how the structures of $n \geq 2$ 2D LHPs evolve as a function of temperature and how the structure is influenced by changing the organic spacer, A-site cation, or quantum-well thickness. This understanding has been hampered, in part, by the difficulty of synthesizing phase-pure variants of $n \geq 2$ 2D LHP single crystals.

2D LHPs can be prepared by a variety of methods. ${ }^{3,21,24,31-35}$ Polycrystalline thin films of 2D LHPs can be made by spin casting a mixture of $\mathrm{PbX}_{2}, \mathrm{AX}$, and $\mathrm{LX}$ dissolved in dimethylformamide and/or dimethylsulfoxide onto a spinning substrate. The same mixture can also be added drop-wise to a non-polar solvent, such as toluene, to produce $2 \mathrm{D}$ nanoplatelets via ligand-assisted reprecipitation. ${ }^{5,7,36}$ Colloidal solutions of $2 \mathrm{D}$ nanoplatelets can be prepared by a modified hotinjection method, ${ }^{37,38}$ similar to the approach used to synthesize halide perovskite quantum dots. ${ }^{39}$ In addition, 2D sheets of LHPs can be grown directly on a substrate by solvent evaporation ${ }^{40}$ as well as chemical vapor deposition. ${ }^{41,42}$ Inverse temperature crystallization, ${ }^{43}$ which employs the retrograde solubility of perovskite reagents, was successfully used to make high-quality single crystals of 3D LHPs. However, the reagents of 2D LHPs do not show retrograde solubility, and the method is not feasible to grow 2D LHP single crystals ${ }^{44}$ due to the difference in surface-solvent interaction. Anti-solvent vapor assisted growth has also been a successful approach for growing 3D LHPs ${ }^{45}$ as well as $n=12$ D LHPs,${ }^{46}$ but successful application of this method to the preparation of $n \geq 2$ 2D LHPs has not been reported (our own attempts were unsuccessful). On the other hand, cooling-induced crystallization has been shown to produce pure single crystals of 2D methylammonium lead iodide perovskites with precise thickness control of up to $n=5 .{ }^{47-49}$ In this 
method, perovskite ions are dissolved in hot aqueous hydrohalic acid, and the solution is then allowed to cool slowly to induce crystallization.

Herein, we report successful cooling-induced crystallization and structural evolution of $\left(\mathrm{C}_{4} \mathrm{H}_{9} \mathrm{NH}_{3}\right)_{2}(\mathrm{MA}) \mathrm{Pb}_{2} \mathrm{I}_{7}, \quad\left(\mathrm{C}_{4} \mathrm{H}_{9} \mathrm{NH}_{3}\right)_{2}(\mathrm{MA})_{2} \mathrm{~Pb}_{3} \mathrm{I}_{10}, \quad\left(\mathrm{C}_{4} \mathrm{H}_{9} \mathrm{NH}_{3}\right)_{2}(\mathrm{MA})_{3} \mathrm{~Pb}_{4} \mathrm{I}_{13}$, $\left(\mathrm{C}_{5} \mathrm{H}_{11} \mathrm{NH}_{3}\right)_{2}(\mathrm{MA}) \mathrm{Pb}_{2} \mathrm{I}_{7},\left(\mathrm{C}_{6} \mathrm{H}_{13} \mathrm{NH}_{3}\right)_{2}(\mathrm{MA}) \mathrm{Pb}_{2} \mathrm{I}_{7}$, and $\left(\mathrm{C}_{4} \mathrm{H}_{9} \mathrm{NH}_{3}\right)_{2}(\mathrm{FA}) \mathrm{Pb}_{2} \mathrm{I}_{7}$. Optical absorption, photoluminescence, and X-ray diffraction further confirmed their chemical compositions and structures. Henceforth, these chemical formulae will be abbreviated as $n=2 \mathrm{BA}-\mathrm{MAPbI}, n=3$ BA-MAPbI, $n=4$ BA-MAPbI, $n=2$ PA-MAPbI, $n=2$ HA-MAPbI, and $n=2$ BA-FAPbI, respectively. Moreover, we discuss factors that influence the phase purity of 2D LHP crystals and provide guidance for achieving 2D LHP crystals of desired composition.

Furthermore, structural dependence of 2D LHPs on quantum-well thicknesses, organic spacer lengths, and A-site cations are discussed. We found that (i) there is a general trend of lower crystal symmetry as the temperature is reduced; (ii) $\mathrm{PbI}$ octahedra on a surface (i.e. proximal to the organic spacer layer) have higher distortion and tilting angles than their middle-layer counterparts; (iii) there is an alternation of axial $\mathrm{Pb}-\mathrm{I}$ bond lengths, with terminal bonds being the shortest; (iv) there is a cohesive trend in the change of $\mathrm{Pb}-(\mu-\mathrm{I})-\mathrm{Pb}$ bond angles as the crystal symmetry is lowered; and (v) there are increases in the extents of interdigitation and corrugation tilts of organic spacer molecules as 2D LHPs adopt their lower-temperature structures. Increasing the length of the organic spacer molecule affects the number of phase transitions of $n=2$ samples and the tilting angles of $\mathrm{PbI}_{6}$ octahedra, while replacing the A-site cation from MA to FA has small effect on the structures - except in the tilting angles of $\mathrm{PbI}_{6}$ octahedra. 
Finally, we conclude by further modification of the cooling-induced crystallization to prepare bromine-based 2D LHPs. Successful syntheses of $n=1 \mathrm{BA}-\mathrm{PbBr}, n=2 \mathrm{BA}-\mathrm{MAPbBr}$, and $n=3$ $\mathrm{BA}-\mathrm{MAPbBr}$ are reported, along with their absorption and photoluminescence spectra and powder X-ray diffraction (PXRD) patterns.

\section{Experimental Section}

Synthesis of iodide 2D LHPs. Crystals of iodide 2D LHPs were synthesized using a modified

procedure based on cooling-induced crystallization method, as reported before. ${ }^{18,47,48}$ For the synthesis of iodide 2D LHPs, a large volume of lead (II) iodide $\left(\mathrm{PbI}_{2}\right)$ was prepared by dissolving lead (II) oxide (PbO, 99.9+\%, (trace metal basis) <10 microns, powder, ACROS Organic) in 55\% hydrogen iodide solution (HI, contains no stabilizer, ACS reagent, 55\%, MilliporeSigma) under reflux. A small volume of this solution was then transferred into a small vial, and mixed with hypophosphorus acid $\left(\mathrm{H}_{3} \mathrm{PO}_{2}\right)$. After the mixed solution turned bright yellow, a small volume of long chain organic spacer $(\mathrm{L}=$ butylamine $\{\mathrm{BA}\}$, pentylamine $\{\mathrm{PA}\}$, or hexylamine $\{\mathrm{HA}\})$ was added, and an orange precipitate of $n=1 \mathrm{~L}-\mathrm{PbI}$ formed. To synthesize a higher member of iodide 2D LHPs, a solution of the A-site cation iodide salt (AI = MAI or FAI) was prepared in a separate vial by dissolving the salt in HI. This AI solution was subsequently added into the solution containing the orange precipitate to form a combined solution. Additional volume of HI may be added before the final solution was heated on a hotplate set at $130{ }^{\circ} \mathrm{C}$ for 4 mins or until clear. Crystals of iodide 2D LHPs were then grown by storing the clear solution in a thermos filled with hot sand at $110{ }^{\circ} \mathrm{C}$. After a day, the crystals were isolated by suction filtration and dried under reduced pressure for at least 12 hours. The quantities of reagents used for making iodide 2D LHPs can be found in Table S1. 
Synthesis of bromide 2 D LHPs. To synthesize bromide 2D LHPs, lead (II) bromide $\left(\mathrm{PbBr}_{2}\right)$ was prepared by dissolving $\mathrm{PbO}$ (99.9+\%, (trace metal basis) < 10 microns, powder, ACROS Organic) in hydrogen bromide solution (HBr, ACS reagent, 48\%, MilliporeSigma)) under reflux. A small volume of this solution was then transferred into a small vial. Without any addition of $\mathrm{H}_{3} \mathrm{PO}_{2}$, a small volume of BA was added and white precipitate of $n=1 \mathrm{BA}-\mathrm{PbBr}$ formed. In a separate vial, a solution of the A-site cation bromide salt $(\mathrm{ABr}=\mathrm{MABr})$ was prepared by dissolving the salt in $\mathrm{HBr}$. This $\mathrm{ABr}$ solution was subsequently added into the solution containing the orange precipitate to form a combined solution. Additional volume of $\mathrm{HBr}$ was added before the final solution was heated on a hotplate set at $130{ }^{\circ} \mathrm{C}$ for 4 mins or until clear. Crystals of bromide 2D LHPs were then grown by storing the clear solution in a thermos filled with hot sand at $110{ }^{\circ} \mathrm{C}$. After a day, the crystals were isolated by suction filtration and dried under reduced pressure for at least 12 hours. The quantities of reagents used for making bromide 2D LHPs can be found in Table S2.

Mechanical exfoliation. Exfoliated flakes of 2D LHPs were prepared using adhesive tapes. A Crystal of 2D LHPs was mounted on a piece of single-sided adhesive tape (Ultron Systems, 1007R6.0). The two ends of the tape were then folded together and separated to exfoliate the crystal. This step was repeated 5-10 times. Thin flakes of 2D LHPs were transferred to a glass substrate by gently pressing the tape to the substrate.

Absorption measurement. Semi-transparent flakes of 2D LHPs were mechanically exfoliated onto fused quartz substrate for absorption measurements. The absorption spectra were measured using transmission mode on a home-built micro-absorption setup. The incident white light beam (Thorlabs, SLS201L) was focused onto the sample through an inverted lens (10X, NA=0.25). The transmitted light was collected by a 100x lens $(\mathrm{NA}=0.90)$ and analyzed by a Raman spectrometer (T64000, Horiba) with $150 \mathrm{~g} / \mathrm{mm}$ grating. The absorbance (A) of a 2D LHP sample was calculated 
by $\mathrm{A}=-\log _{10}\left(\mathrm{I} / \mathrm{I}_{0}\right)$, where $\mathrm{I}$ and $\mathrm{I}_{0}$ are the transmitted light intensities on the $2 \mathrm{D}$ LHP flake and nearby quartz substrate.

Photoluminescence. Steady-state photoluminescence measurements were performed on an inverted microscope (Nikon, Ti-U Eclipse) in air or vacuum. The samples were excited by focusing the output of a $405 \mathrm{~nm}$ pulsed laser diode (Picoquant, LDHDC-405M, 2.5 MHz repetition rate) by an objective lens (Nikon, CFI S Plan Fluor ELWD, 40x, $0.6 \mathrm{NA}$ ) to $<1 \mu \mathrm{m}$ spot. The photoluminescence was collected in the epi configuration, and passed through a dichroic mirror and a long-pass filter. Then, it was directed into a spectrograph (SP-2500, Princeton Instruments) mounted with a cooled charge-coupled detector (Princeton Instruments, Pixis). Laser power was kept below $\sim 125 \mathrm{nW}$ throughout the measurements. For the measurement on $n=1 \mathrm{BA}-\mathrm{PbBr}$, the excitation source was replaced by a 375 nm LED (Thorlabs, M375F2). Photoluminescence measurements under vacuum were achieved by mounting 2D LHP crystals in a microscopy cryostat (Janis Research, ST-500). Photoluminescence spectra measured under air and vacuum were identical.

Powder X-ray diffraction (PXRD). Powder X-ray diffraction data was collected using a PANalytical X'Pert Pro MPD X-ray diffractometer $(\mathrm{Cu}$ K $\alpha$ radiation, $\lambda=1.54184 \AA)$ with HighSpeed Bragg-Brentano Optics. A 0.04 rad soller slit, a $1^{\circ}$ anti-scatter slit, a $10 \mathrm{~mm}$ mask and a programmable divergence slit with an illuminated length of $6 \mathrm{~mm}$ were used in the incident beam path. The diffracted beam optics included a $0.04 \mathrm{rad}$ soller slit, a Ni Filter and an automatic receiving slit. The detector was an ultrafast $\mathrm{X}^{\prime}$ Celerator RTMS detector. The angular step in $2 \theta$ was $0.04^{\circ}$. 
Single-crystal X-ray crystallography. A crystal was mounted on a diffractometer at $300 \mathrm{~K}$. The temperature was raised/lowered down to measurement temperatures at a rate of $5 \mathrm{~K} / \mathrm{h}$ before data sets were collected. The intensities of the reflections were collected by means of a Bruker APEX II CCD diffractometer (Мoк $\alpha$ radiation, $\lambda=0.71073 \AA$ ), and equipped with an Oxford Cryosystems nitrogen flow apparatus. The collection method involved $0.5^{\circ}$ scans in $\omega$ at $28^{\circ}$ in

20. Data integration down to $0.84 \AA$ A resolution was carried out using SAINT V8.37A (Bruker diffractometer, 2016) with reflection spot size optimization. Absorption corrections were made with the program SADABS (Bruker diffractometer, 2016). The structure was solved by the Intrinsic Phasing methods and refined again by least-squares methods $F^{2}$ using SHELXT-2014 ${ }^{50}$ and SHELXL-2014 ${ }^{51}$ with OLEX 2 interface. ${ }^{52}$ Non-hydrogen atoms were refined anisotropically, and hydrogen atoms were allowed to ride on the respective atoms. Crystal data as well as details of data collection and refinement can be found in the supporting information. Further details on refinement are also available in the CIF files provided. Visualization of crystallographic data was performed by using VESTA. ${ }^{53}$

\section{Results and Discussion}

\section{Syntheses of iodide 2D LHPs}

Iodide 2D LHPs were synthesized using a modified procedure based on the cooling-induced crystallization method ${ }^{18,47,48}$ (Figure 1a). Detailed tables specifying reagent quantities and conditions used to synthesize each 2D LHP composition can be found in the Supporting Information. Additional procedural details are included in the experimental section. Briefly, a large volume of lead (II) iodide $\left(\mathrm{PbI}_{2}\right)$ solution was prepared by the reaction of lead (II) oxide $(\mathrm{PbO})$ and 55\% hydrogen iodide (HI) solution under reflux. A small volume of $\mathrm{PbI}_{2}$ solution was then 
transferred into a vial, and hypophosphorus acid $\left(\mathrm{H}_{3} \mathrm{PO}_{2}\right)$ was added to reduce any residual iodine ( $\mathrm{I}_{2}$ ) back to HI. After a few seconds, the solution turned bright yellow. Next, the long chain organic cation (L) was added, forming an orange precipitate of $n=1 \mathrm{~L}-\mathrm{PbI}$. To make thicker members of the iodide 2D LHP family $(n>1)$, a solution of the A-site cation iodide (AI) salt was prepared in a separate vial by dissolving the salt into HI. This AI solution was subsequently added into the solution containing the orange precipitate. This combined solution was then heated until clear, and slowly cooled down to produce crystals of iodide 2D LHPs. The crystals were then collected by suction filtration and dried under reduced pressure. Photographs of crystals obtained by this method are shown in Figure 1b.

We found that controlling relative quantities of reagents was key to obtaining pure crystals. Deviating from the optimal concentrations in the starting solutions resulted in crystals containing impurities of thicker (e.g. $n+1$ ) and/or thinner (e.g. $n$ - 1) iodide 2D LHPs. Because of the differences in solubilities among different reagents, each solidifies at a different rate and at different temperature during cooling. Consequently, the optimal starting concentrations are not simply the stoichiometric amounts found from the chemical formulae of iodide 2D LHPs; to obtain pure crystals of the desired iodide 2D LHPs, the precipitation rates of precursors must be balanced, which can be achieved by adjusting concentrations.

The overall effect of changing long chain organic spacer concentrations can be predicted from the chemical formulae of iodide 2D LHPs. As we go from $n=1$ iodide 2D LHP to bulk LHP, the L:Pb ratio decreases from 2:1 to $0: 1$. This suggests that the higher concentration of long chain organic cation favors the formation of thinner (lower $n$ ) iodide 2D LHPs, which was indeed observed experimentally (Figure S1). The long chain organic spacer is the least soluble component of the reaction mixture and is the first to precipitate as the solution is cooled. When a stoichiometric 
amount of long chain organic spacer was used, a complete crystallization of orange $n=1$ iodide 2D LHP or thinner (i.e. $n-1$ ) iodide 2D LHPs was observed. Consequently, the amount of long chain organic spacer used in our synthesis was always kept below those predicted from stoichiometry (Table S1).
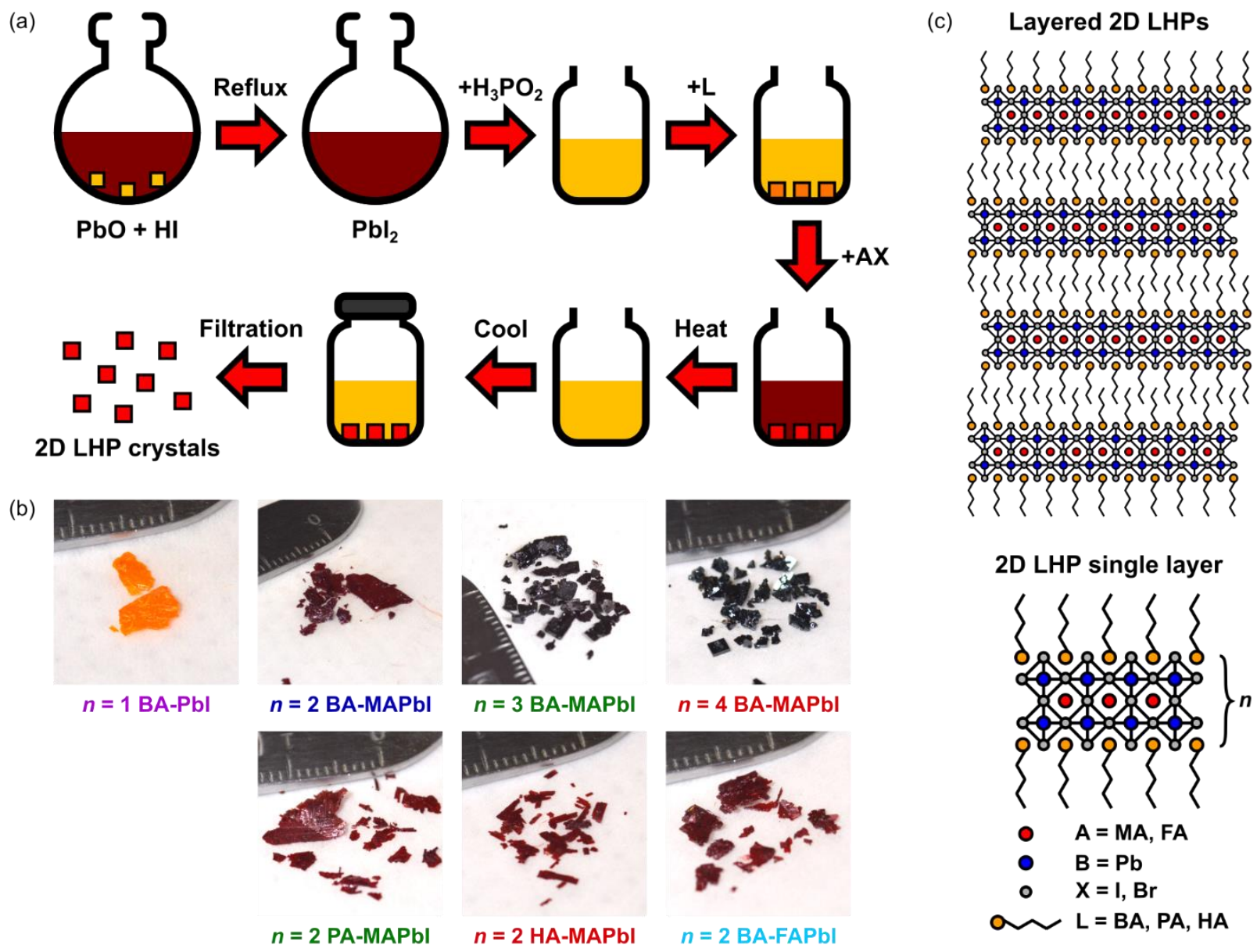

Figure 1. (a) Schematic describing cooling-induced crystallization of 2D lead halide perovskites (LHPs). (b) Photographs of 2D LHP crystals and corresponding illustration of the 2D LHP structure. The spacing between minor ticks on the ruler is $1 \mathrm{~mm}$. (c) Schematic illustrations showing the structures of layered 2D LHPs and a 2D LHP single layer. MA = methylammonium; $\mathrm{FA}=$ formamidinium; $\mathrm{BA}=$ butylammonium; $\mathrm{PA}=$ pentylammonium; $\mathrm{HA}=$ hexylammonium .

Because the solubility of the long chain organic spacer in HI decreases with increasing alkylammonium chain length, decreasing concentrations are needed as the chain length increases. 
The percentages of long chain organic spacers used with respect to their stoichiometric amounts for making $n=2 \mathrm{BA}-\mathrm{MAPbI}, n=2 \mathrm{PA}-\mathrm{MAPbI}$, and $n=2 \mathrm{HA}-\mathrm{MAPbI}$ decreased from $65 \%$ to $60 \%$ to $50 \%$, respectively (Table S1). The trend in solubility also affects the crystal shape. As HA is the least soluble among the three organic spacers, it precipitated out fastest during cooling resulting in thinner, smaller crystals. On the other hand, large crystals with lateral sizes of up to 5 mm of $n=2$ BA-MAPbI and $n=2$ PA-MAPbI (Figure $1 \mathrm{~b}$ ) could be obtained easily with this method. These crystals were large enough that lamellar structure is evident in macro-scale morphology. This observation agrees with the reflections along (h00) planes in X-ray diffraction data as reported previously ${ }^{18,44}$ and shown again in this work.

Thicker (higher- $n$ ) 2D LHPs can be obtained by decreasing the concentration of the long chain organic spacer in the reaction mixture. The optimal percentage of long chain organic spacer with respect to the stoichiometrically required amount shows a downward trend (Table S1) due to higher solubility of the A-site cation and lower solubility of long chain organic spacer. We found that fixing the percentage of long chain organic spacer while changing $n$ results in thinner (e.g. $n$ - 1) impurities.

The effect of changing A-site cation concentration can be understood similarly. We observed that increasing the concentration of the A-site cation favors the formation of thicker (higher- $n$ ) iodide 2D LHPs (Figure S2). In most of our syntheses, stoichiometric amounts of A-site cations were used. However, due to the low solubility of HA, a more saturated solution of MA (more MA in the starting mixture) was needed to match the precipitation rate of HA. 175\% of MA stoichiometric amount was needed to form phase-pure $n=2 \mathrm{HA}-\mathrm{MAPbI}$. When a stoichiometric amount of MA was used, orange crystals of $n=1$ iodide 2D LHP were observed to be the main products, regardless of the initial concentration of HA (Figure S3). 
Adjusting the total volume of $\mathrm{HI}$ also affected the quality of the final crystallization product. We found that a small volume of HI often resulted in mixed-phase iodide 2D LHPs and crowded solutions with small crystals, whereas increasing HI volume promoted the formation of larger crystals. However, when too much HI was added the A-site cation did not precipitate out of solution, resulting in the formation of $n=1$ iodide 2D LHP crystals (Figure S4). We also found that greater $\mathrm{HI}$ volume (per gram of $\mathrm{PbO}$ ) was needed for the weakly-soluble longer-chain organic spacers. When tuning $n$, HI volume was kept the same to minimize the number of changing parameters. Detailed tables specifying reaction conditions and reagent quantities can be found in the Supporting Information.

When the A-site cation was changed from MA to FA or Cs, additional difficulties emerged. In the case of FA cation, the precipitation rate was found to be faster, and the resulting crystal sizes tended to be smaller. To grow larger crystals of high-purity $n=2 \mathrm{BA}-\mathrm{FAPbI}$, the volume of $\mathrm{HI}$ was increased relative to the amounts of other reagents. Attempts to synthesize $n>2$ BA-FAPbI were unsuccessful; formation of $n=2 \mathrm{BA}-\mathrm{FAPbI}$ was always observed, regardless of how the synthesis conditions were varied (Figure S5). This observation suggests that $n=2 \mathrm{BA}-\mathrm{FAPbI}$ might be the most thermodynamically stable product among the iodide 2D LHPs with FA cation. ${ }^{3}$ Similar observations have been reported in the case of $n=5$ BA-MAPbI, which undergoes disproportionation to form $n=3 \mathrm{BA}-\mathrm{MAPbI}$ and bulk MAPbI $3 .{ }^{48}$ Finally, we note that attempts to synthesize iodide 2D LHPs with Cs cation were unsuccessful due to the poor solubility of CsI in HI. These attempts resulted in a mixture of white CsI solid and orange $n=1$ BA-PbI 2D LHP (Figure S5). 
In general, we observed some seasonal variation in the synthesis product. During high-humidity seasons, needle-liked crystals with yellow color emerged as a by-product (Figure S6). However, their formation could be avoided by slightly increasing the AX fraction by approximately $5 \%$.

\section{Stability of iodide 2D LHP crystals and exfoliated flakes:}

All of the iodide 2D LHP crystals synthesized by this method were stable to storage inside a $\mathrm{N}_{2}$ glovebox for more than one year (Figure S7). Additionally, the large crystals shown in Figure 1 could be mechanically exfoliated to form thin 2D LHP flakes (Figure S11; see Experimental Section). However, when the crystals were exfoliated and kept under ambient conditions, newly generated surfaces underwent a transition after $\sim 3$ days to form a yellow solid (Figure S8). We hypothesize that, because the interaction between the long chain organic spacer and the lead halide octahedra has a hydrogen-bonding character, ${ }^{54}$ the long chain organic spacer can be easily stripped off during exfoliation. The newly exposed surface is unprotected, and susceptible to attack from $\mathrm{H}_{2} \mathrm{O}$ and $\mathrm{O}_{2}$ in the air to form $\mathrm{PbI}_{2}$. This hypothesis was tested by storing exfoliated crystals either in inert atmosphere or in oil. No apparent color change was observed under either condition (In the case of oil, the experiment was performed for one week). These observations suggest that the stability of exfoliated 2D LHP flakes could be further improved through encapsulation by a polymer layer ${ }^{55,56}$ or another 2D material, such as hexagonal boron nitride $(\mathrm{hBN}) .55,57-59$

\section{Optical characterization of iodide 2D LHP crystals:}

Room temperature absorption and photoluminescence spectra of exfoliated iodide 2D LHPs are shown in Figure 2. All variants exhibit a single sharp excitonic absorption resonance 100-500 meV below the continuum band and Stokes-shifted emission close in energy to the 1s excitonic absorption feature. 2D alkylammonium LHPs exhibit large exciton binding energies (470 meV for 
$n=1$ butylammonium lead iodide) due to spatial confinement of electrons and holes within the 2D inorganic lattice, and low dielectric screening from the long chain organic spacer layer. ${ }^{6}$ Increasing $n$ red-shifts the excitonic resonance in both absorption (Abs) and photoluminescence (PL) spectra (Figure 2a). These shifts are the result of two partially offsetting factors: quantum confinement, which widens the electronic band gap as $n$ decreases, and dielectric confinement, which leads to an increase in exciton binding energy as $n$ decreases - thereby reducing the magnitude of the overall blue-shift. The $n=2-4$ samples show similar photoluminescence (PL) full width at half maximum (FWHM) of $25-27 \mathrm{~nm}$, as well as similar Stokes shifts of $\sim 10 \mathrm{~nm}$ (Table S3). In contrast, the $n=1$ sample shows narrower PL FWHM of $17 \pm 2 \mathrm{~nm}$.

For the $n=2$ iodide samples, Abs and PL spectra were largely unaffected when the organic spacer length increased from four to six carbons (Figure 2b). All samples showed Abs peaks centered near $\sim 570 \mathrm{~nm}$, PL peaks centered near $\sim 580 \mathrm{~nm}$, PL FWHM of $\sim 25 \mathrm{~nm}$ and Stokes shift of $\sim 10$ $\mathrm{nm}$ (Table S3). However, when the A-site cation was changed from MA to FA (Figure 2c), the PL FWHM decreased from $25 \pm 2 \mathrm{~nm}$ to $21 \pm 2 \mathrm{~nm}$.

The observation of single Abs and PL peaks in each sample also indicates the high purity of these samples. Occasionally, when checking PL from multiple locations under a microscope, regions exhibiting PL from thicker (e.g. $n+1)$ or thinner (e.g. $n-1)$ iodide 2D LHPs could be observed. These observations were rare in high-purity samples (Figure S9), and phase impurity was never detected by powder X-ray diffraction (next section). 
(a)

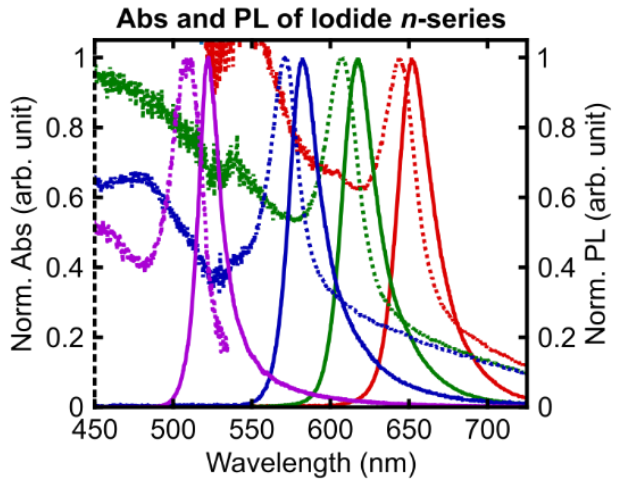

$$
\begin{aligned}
& -n=1 \text { BA-Pbl }-n=3 \text { BA-MAPbI } \\
& -n=2 \text { BA-MAPbI }-n=4 \text { BA-MAPbI }
\end{aligned}
$$

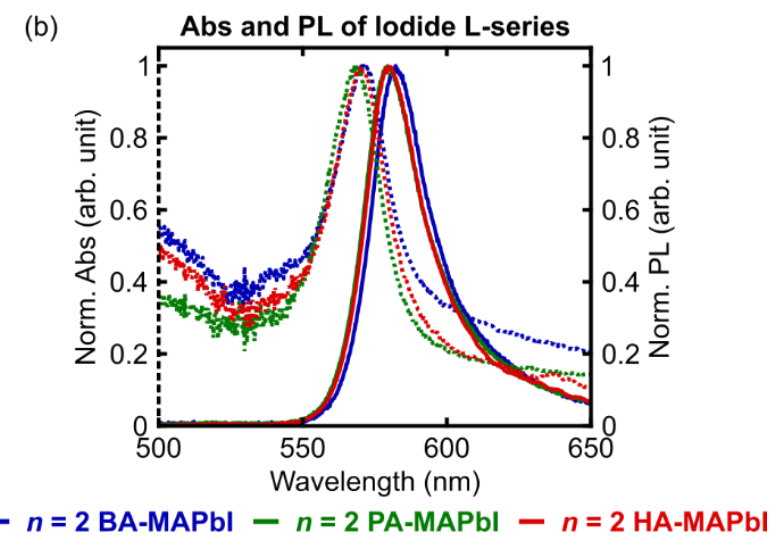

(c)

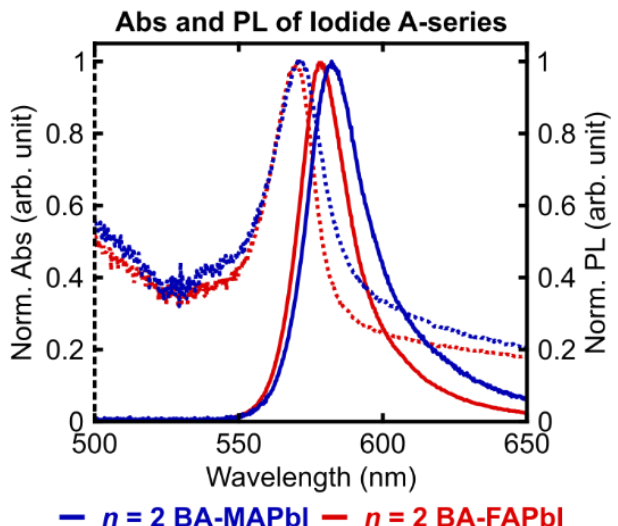

Figure 2. Absorption (dotted) and photoluminescence (solid) spectra of exfoliated 2D LHP flakes at room temperature. Comparisons are shown for (a) $n=1 \mathrm{BA}-\mathrm{PbI}, n=2 \mathrm{BA}-\mathrm{MAPbI}, n=3 \mathrm{BA}$ MAPbI, and $n=4 \mathrm{BA}-\mathrm{MAPbI}$; (b) $n=2 \mathrm{BA}-\mathrm{MAPbI}, n=2 \mathrm{PA}-\mathrm{MAPbI}$, and $n=2 \mathrm{HA}-\mathrm{MAPbI}$; (c) $n=2 \mathrm{BA}-\mathrm{MAPbI}$ and $n=2 \mathrm{BA}-\mathrm{FAPbI}$. 


\section{Structural insights from powder X-ray diffraction}

Initial structural characterization was performed using powder X-ray diffraction (PXRD) on finely ground crystals (Figure 3a-c). All samples showed (111) peaks near $14^{\circ} 2 \theta$ angle and characteristic stacking peaks of 2D layered materials, which manifest as periodic (h00) diffraction peaks in the

PXRD pattern. ${ }^{47}$ Below the (111) peak, we observe either two, three, four, or five of the evenly spaced (h00) reflections for samples with BA organic spacer and $n=1,2,3$, and 4 respectively (Figure $3 a) .{ }^{47}$ The increasing number of reflections in this region indicates a larger unit cell parameter along the stacking direction, and this number can be used to confirm the value of $n$ and the purity of the samples. When the organic spacer is lengthened from four to five and six carbons, the evenly spaced reflections shift to lower $2 \theta$ angles (Figure $3 \mathrm{~b}$ ), reflecting the larger spacing distances in samples with longer alkylammonium chains. The substitution of A-site cation from MA to FA on the other hand does not induce a significant change in the PXRD patterns as shown in Figure 3c.

Using the positions of (h00) reflections, the stacking period of each iodide 2D LHP can be calculated. The stacking periods of $n=1 \mathrm{BA}-\mathrm{PbI}, n=2 \mathrm{BA}-\mathrm{MAPbI}, n=3 \mathrm{BA}-\mathrm{MAPbI}$, and $n=4$ BA-MAPbI were deduced to be $1.39 \mathrm{~nm}, 1.96 \mathrm{~nm}, 2.60 \mathrm{~nm}$, and $3.22 \mathrm{~nm}$, respectively. This incremental increase of $\sim 0.6 \mathrm{~nm}$ corresponds to the thickness of an additional $\mathrm{PbI}$ octahedral layer as $n$ increases. ${ }^{18,47}$ Using the same approach, stacking periods of $n=2$ PA-MAPbI and $n=2$ HAMAPbI were found to be $2.07 \mathrm{~nm}$ and $2.25 \mathrm{~nm}$, larger than that of $n=2$ BA-MAPbI by $0.11 \mathrm{~nm}$ and $0.29 \mathrm{~nm}$, respectively. This non-stepwise increase as the alkylammonium chain length increases is due to the different extents of interdigitation in the organic spacer layers as the organic spacer length is changed, as determined by single crystal X-ray diffraction (SCXRD - see following section). When changing the A-site cation from MA to FA, the stacking period remained 
at a constant $1.96 \mathrm{~nm}$. This is not surprising, since MA and FA have similar ionic radii of $2.70 \AA$ and $2.79 \AA$, respectively. ${ }^{60}$

In addition to the shifts in the stacking peaks, changing sample identities can lead to a similar effect on the position of the (111) peak. As the period of the (111) planes is influenced by the unit cell parameter in the stacking direction, increasing $n$ results in the (111) peaks moving to lower $2 \theta$ angles (Figure 3a). When the organic spacer molecule is changed from BA to PA and HA, the (111) peak starts to split to two peaks of (111) and (11) reflections (Figure 3b). This split arises from the fact that $n=2 \mathrm{BA}-\mathrm{MAPbI}, n=2 \mathrm{PA}-\mathrm{MAPbI}$, and $n=2 \mathrm{HA}-\mathrm{MAPbI}$ have different crystal symmetries: $n=2$ BA-MAPbI adopts orthorhombic $C m c m$, but $n=2$ PA-MAPbI and $n=2$ HAMAPbI adopt monoclinic $C 2 / c$ at room temperature. Similar to the effect on the stacking peaks, replacing MA by FA has a marginal effect on the (111) peak position (Figure 3c). 
(a)

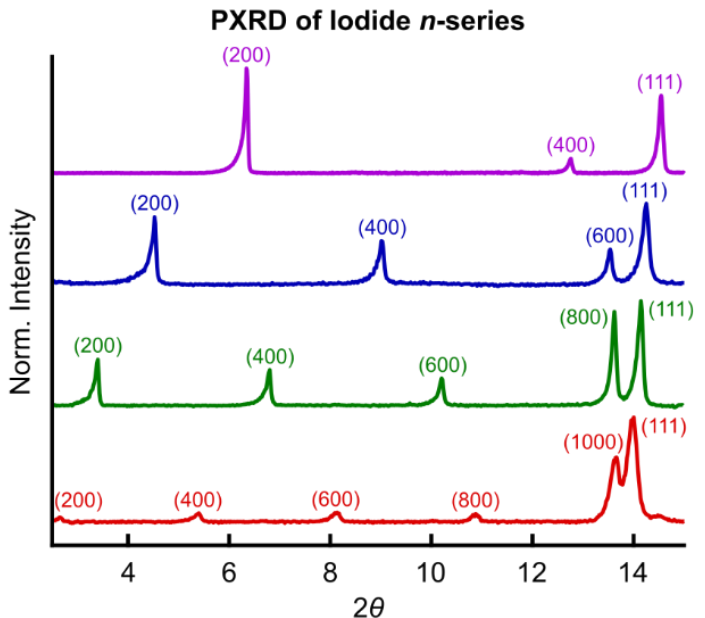

$$
\begin{array}{ll}
-n=1 \text { BA-Pbl } & -n=3 \text { BA-MAPbI } \\
-n=2 \text { BA-MAPbI } & -n=4 \text { BA-MAPbI }
\end{array}
$$

(b)

PXRD of lodide L-series

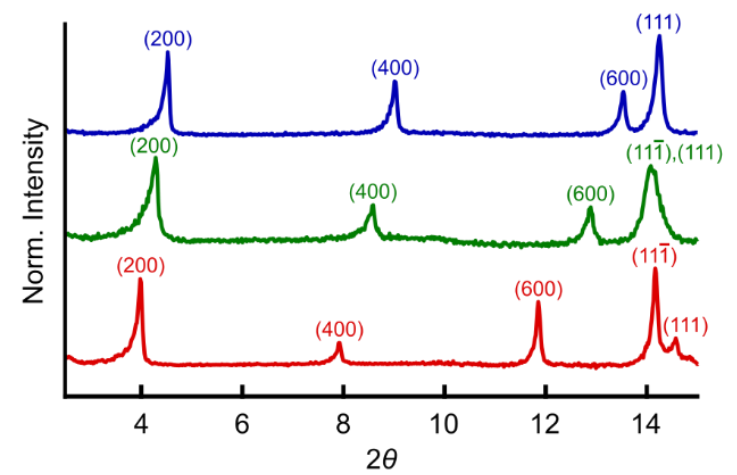

$-n=2$ BA-MAPbI $-n=2$ PA-MAPbI $-n=2$ HA-MAPbI

(c)

PXRD of lodide A-series

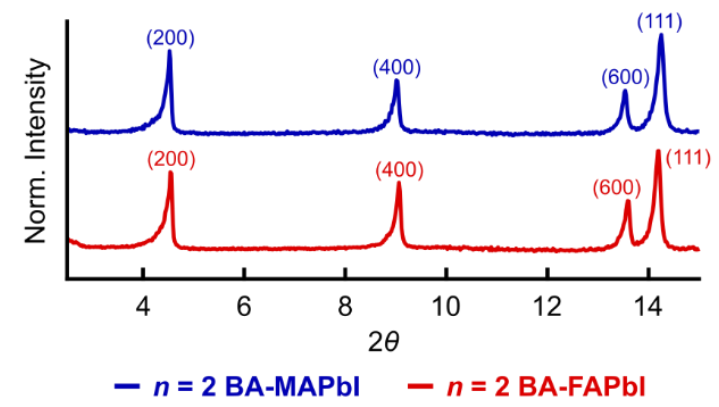

Figure 3. Powder X-ray diffractograms (PXRD) of (a) iodide $n$-series, (b) iodide L-series, and (c) iodide A-series. All peaks are labelled with their corresponding diffraction planes. Evenly spaced (h00) peaks correspond to the periodicity in the stacking direction. 


\section{Analysis of iodide 2D LHP crystal structures}

Crystal structures of all iodide 2D LHP samples were acquired by performing SCXRD on single crystals obtained directly from their mother liquors. The structures of each iodide 2D LHP sample consists of stacks of inorganic slabs separated by bilayers of organic molecular spacers. In the inorganic sublattice, $\mathrm{PbI}_{6}$ octahedra share their corners in both lateral and stacking directions, and the number of $\mathrm{PbI}_{6}$ octahedra layers increase with $n$. The inorganic sublattice shows distortion with respect to the perfect cubic unit cell of the iodide 3D LHP, and adjacent PbI6 octahedra have outof-phase out-of-plane tilting with respect to each other. In all samples, adjacent inorganic slabs are staggered, and all samples are categorized as members of Ruddlesden-Popper perovskites. ${ }^{3,47}$

\section{- 5.1 Structural comparison of iodide 2D LHP crystals with different $n$}

At $300 \mathrm{~K}, n=2,3$, and 4 BA-MAPbI crystallizes in orthorhombic space groups $\mathrm{Cmcm}$, Cmca, and Cmcm, respectively (Figure 4). For $n=2 \mathrm{BA}-\mathrm{MAPbI}$, all $\mathrm{PbI}_{6}$ octahedra are equivalent, and can be related by symmetry operations. When the quantum-well thickness increases to $n=3$ and 4 , two distinct types of $\mathrm{PbI}_{6}$ octahedra emerge: surface $\mathrm{PbI}_{6}$ octahedra, which are adjacent to the organic spacer layer, and middle-layer PbI6 octahedra, which are surrounded on all sides by other PbI6 octahedra. Using this terminology, all PbI6 octahedra in $n=2$ iodide 2D LHPs can be treated as surface $\mathrm{PbI}_{6}$ octahedra. The surface and the middle-layer $\mathrm{PbI}_{6}$ octahedra differ by their out-ofplane tilting angles. The out-of-plane tilting angles of the $\mathrm{Pb}-\mathrm{I}$ bonds on the surfaces are between $9.5^{\circ}$ and $10^{\circ}$, and for the non-surface $\mathrm{Pb}$-I bonds, these numbers decreases to $\sim 6-7^{\circ}$ in $n=2$ and 4 samples, and $0^{\circ}$ in $n=3$ sample (Figure S12). Although the middle-layer of $n=3$ BA-MAPbI does not have any out-of-plane tilting, it still showed distortion in the form of in-plane, out-of-phase rotation between adjacent PbI6 octahedra. The difference in out-of-plane tilting angles in the 
surface $\mathrm{PbI}_{6}$ octahedra and the middle-layer $\mathrm{PbI}_{6}$ octahedra reflect the difference in the interaction between the $\mathrm{PbI}_{6}$ octahedral framework with the smaller MA cation and the larger organic spacer cations.

In addition to the out-of-plane tilting, the degree of distortion can be quantified by bond length distortion $^{61-64}$ and bond angle variance. ${ }^{61,65}$ The bond length distortion $(\Delta d)$ of an octahedron is defined as

$$
\Delta d=\left(\frac{1}{6}\right) \sum\left[\frac{d_{n}-d}{d}\right]^{2}
$$

where $d_{n}$ is the individual $\mathrm{Pb}-\mathrm{I}$ bond distance and $d$ is the mean $\mathrm{Pb}$-I bond distance. Additionally, the bond angle variance $\left(\sigma^{2}\right)$ of an octahedron can be calculated by

$$
\sigma^{2}=\sum_{i=1}^{12} \frac{\left(\theta_{i}-90\right)^{2}}{11}
$$

where $\theta_{i}$ is the individual I-Pb-I angle. Nonzero values of these two parameters indicate distortion from a perfect octahedron, which is characterized by six equal bond lengths and $90^{\circ}$ bond angles. Across $n=2,3$, and 4 BA-MAPbI, both $\Delta d$ and $\sigma^{2}$ are larger for surface octahedra than in middlelayer octahedra (Figure 4d left panel), in agreement with the extent of out-of-plane tilting discussed above.

A closer inspection of the bond lengths across samples with different $n$ also sheds light on how bonding orbitals of $\mathrm{Pb}$ and I interact. In iodide 2D LHPs, $\mathrm{Pb}$-I bonds can be classified as terminalaxial (T-Ax) Pb-I bonds, linking-axial (L-Ax) Pb-I bonds, and linking-equatorial (L-Eq) Pb-I bonds (Figure S13), where axial and equatorial directions are defined as out-of-plane and in-plane directions, respectively. A general trend observed across all iodide 2D LHP samples is that the T- 
$\mathrm{Ax} \mathrm{Pb}-\mathrm{I}$ bond is always the shortest bond among the three types. This can be explained by noting that the orbitals of a terminal I atom are only interacting with the orbitals from one $\mathrm{Pb}$ atom, whereas those of a linking I atom are shared with the orbitals from two $\mathrm{Pb}$ atoms (Figure 4e). Hence, orbital overlap is greatest in the $\mathrm{T}-\mathrm{Ax} \mathrm{Pb}-\mathrm{I}$ bond, and this bond is the shortest and strongest. Going from the top to the bottom of the inorganic slab, the axial $\mathrm{Pb}-\mathrm{I}$ bond lengths exhibit a zigzag pattern with a reflection plane at the middle of the inorganic slab (Figure 4f). This behavior can be explained by noting that the two Ax I atoms in each $\mathrm{PbI}_{6}$ octahedron are interacting with the same orbitals of $\mathrm{Pb}$ atom (Figure 4e). Stronger orbital overlap in one $\mathrm{Ax} \mathrm{Pb}$-I bond will thus make the orbital overlap of the other $\mathrm{Ax} \mathrm{Pb}-\mathrm{I}$ bond weaker, resulting in two $\mathrm{Ax} \mathrm{Pb}-\mathrm{I}$ bonds with different bond lengths. This effect is called the trans effect, and is commonly found in transition metal complexes. ${ }^{66}$ This alternation of bond length is not unique to 2D LHPs, and has been previously observed in both cubic and tetragonal phases of $\mathrm{MAPbI}_{3}{ }^{67}$ The $\mathrm{Pb}-\mathrm{I}$ bond lengths in cubic and tetragonal $\mathrm{MAPbI}_{3}$ alternate between $3.21 \AA$ and $3.10 \AA$ for the former and between $3.20 \AA$ and $3.13 \AA$ for the latter, which are similar to those found in the middle-layer PbI6 octahedra $(3.19 \AA$ and $3.12 \AA$ ). However, an exception of bond-length alternation was observed in the middle-layer $\mathrm{PbI}_{6}$ octahedra of $n=3 \mathrm{BA}-\mathrm{MAPbI}$ which has equal Ax Pb-I bond lengths due to symmetry. On the other hand, L-Eq Pb-I bond lengths have a small variation, and are 3.16 $\pm 0.02 \AA$ long, which is similar to those found in cubic and tetragonal $\mathrm{MAPbI}_{3}{ }^{67}$ 
Table 1. Crystal data and structure refinement for $n=2 \mathrm{BA}-\mathrm{MAPbI}, n=3 \mathrm{BA}-\mathrm{MAPbI}$, and $n=$ 4 BA-MAPbI

\begin{tabular}{|c|c|c|c|c|c|c|}
\hline & \multicolumn{2}{|c|}{$n=2 \mathrm{BA}-\mathrm{MAPbI}$} & \multicolumn{2}{|c|}{$n=3 \mathrm{BA}-\mathrm{MAPbI}$} & \multicolumn{2}{|c|}{$n=4 \mathrm{BA}-\mathrm{MAPbI}$} \\
\hline Chemical formula & \multicolumn{2}{|c|}{$\mathrm{C}_{9} \mathrm{H}_{30} \mathrm{I}_{7} \mathrm{~N}_{3} \mathrm{~Pb}_{2}$} & \multicolumn{2}{|c|}{$\mathrm{C}_{10} \mathrm{H}_{36} \mathrm{I}_{10} \mathrm{~N}_{4} \mathrm{~Pb}_{3}$} & \multicolumn{2}{|c|}{$\mathrm{C}_{11} \mathrm{H}_{42} \mathrm{I}_{13} \mathrm{~N}_{5} \mathrm{~Pb}_{4}$} \\
\hline$M_{\mathrm{r}}$ & \multicolumn{2}{|c|}{1483.04} & \multicolumn{2}{|c|}{2103.00} & \multicolumn{2}{|c|}{2722.95} \\
\hline Temperature (K) & 300 & 250 & 300 & 250 & 300 & 250 \\
\hline Crystal system & Orthorhombic & Triclinic & Orthorhombic & Triclinic & Orthorhombic & Triclinic \\
\hline Space group & $\mathrm{Cmcm}$ & $P-1$ & Cmca & $P-1$ & $\mathrm{Cmcm}$ & $P-1$ \\
\hline$a(\AA)$ & 39.277 (4), & $8.7432(10)$ & $51.963(8)$ & $8.7799(13)$ & $64.305(5)$ & $8.8047(11)$ \\
\hline$b(\AA)$ & $8.9317(8)$ & $9.0448(10)$ & $8.9349(13)$ & $9.0236(14)$ & $8.9327(6)$ & $8.9991(12)$ \\
\hline$c(\AA)$ & $8.8533(8)$ & 19.669 (2) & $8.8648(13)$ & 25.436 (4) & $8.8835(6)$ & $31.917(4)$ \\
\hline$\alpha\left(^{\circ}\right)$ & 90 & $102.905(3)$ & 90 & $90.030(3)$ & 90 & $97.912(2)$ \\
\hline$\beta\left(^{\circ}\right)$ & 90 & $95.093(3)$ & 90 & $95.685(3)$ & 90 & $93.216(2)$ \\
\hline$\gamma\left({ }^{\circ}\right)$ & 90 & $91.393(3)$ & 90 & $91.316(3)$ & 90 & $91.222(2)$ \\
\hline$V\left(\AA^{3}\right)$ & $3105.9(5)$ & $1508.6(3)$ & $4115.8(10)$ & $2004.8(5)$ & $5102.9(6)$ & $2499.9(6)$ \\
\hline$Z$ & 4 & 2 & 4 & 2 & 4 & 2 \\
\hline$\mu\left(\mathrm{mm}^{-1}\right)$ & 17.79 & 18.31 & 19.75 & 20.27 & 21.04 & 21.47 \\
\hline Crystal size (mm) & $\begin{array}{l}0.10 \times 0.06 \times \\
0.02\end{array}$ & $\begin{array}{l}0.10 \times 0.06 \\
\times 0.02\end{array}$ & $\begin{array}{l}0.18 \times 0.09 \times \\
0.06\end{array}$ & $\begin{array}{l}0.18 \times 0.09 \\
\times 0.06\end{array}$ & $\begin{array}{l}0.12 \times 0.08 \times \\
0.02\end{array}$ & $\begin{array}{l}0.12 \times 0.08 \\
\times 0.02\end{array}$ \\
\hline $\begin{array}{l}\text { No. of measured } \\
\text { reflections }\end{array}$ & 24804 & 25836 & 27562 & 28054 & 34882 & 34039 \\
\hline $\begin{array}{l}\text { No. of independent } \\
\text { reflections }\end{array}$ & 1506 & 5344 & 1859 & 7091 & 2459 & 8853 \\
\hline $\begin{array}{l}\text { No. of observed }[I> \\
2 \sigma(I)] \text { reflections }\end{array}$ & 1100 & 4101 & 1428 & 5876 & 1677 & 6378 \\
\hline$R_{\text {int }}$ & 0.053 & 0.052 & 0.045 & 0.042 & 0.049 & 0.044 \\
\hline$(\sin \theta / \lambda)_{\max }\left(\AA^{-1}\right)$ & 0.596 & 0.597 & 0.597 & 0.597 & 0.597 & 0.596 \\
\hline $\begin{array}{l}R\left[F^{2}>2 \sigma\left(F^{2}\right)\right] \\
w R\left(F^{2}\right), S\end{array}$ & $\begin{array}{l}0.039,0.101 \\
1.07\end{array}$ & $\begin{array}{ll}0.057, \\
0.138, & 1.08\end{array}$ & $\begin{array}{l}0.042,0.115 \\
1.09\end{array}$ & $\begin{array}{l}0.056, \\
0.136,1.09\end{array}$ & $\begin{array}{l}0.041,0.109 \\
1.05\end{array}$ & $\begin{array}{l}0.062, \\
0.166,1.07\end{array}$ \\
\hline No. of reflections & 1506 & 5344 & 1859 & 7091 & 2459 & 8853 \\
\hline No. of parameters & 92 & 222 & 109 & 276 & 133 & 355 \\
\hline No. of restraints & 71 & 126 & 68 & 124 & 84 & 113 \\
\hline$\Delta \rho_{\max }, \Delta \rho_{\min }\left(\mathrm{e} \AA^{-3}\right)$ & $1.32,-0.93$ & $2.18,-1.56$ & $0.62,-2.00$ & $2.21,-4.06$ & $1.11,-1.98$ & $2.57,-3.98$ \\
\hline
\end{tabular}


(a)

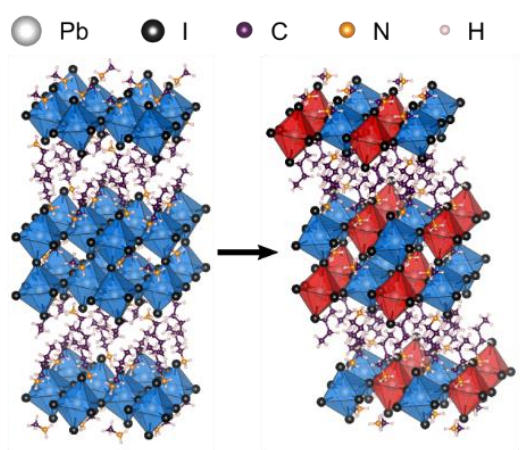

(b)

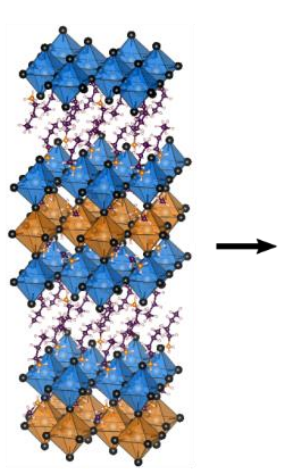

$n=3$ BA-MAPbI

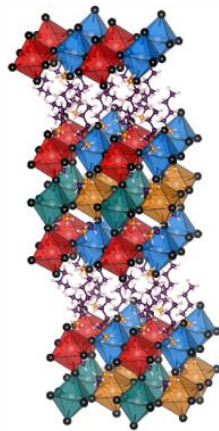

(c)

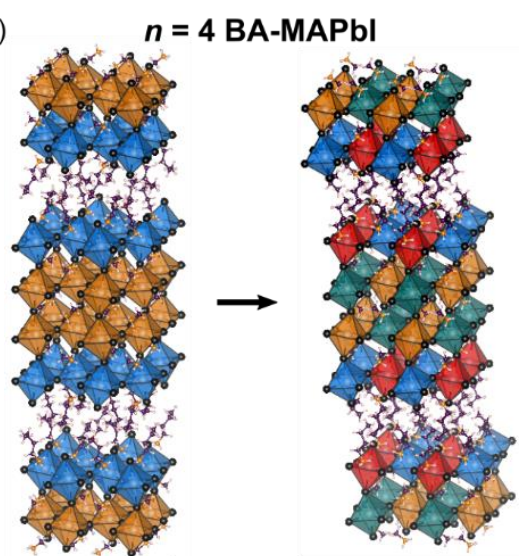

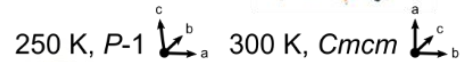

$250 \mathrm{~K}, P-1 \stackrel{\mathrm{L}}{\mathrm{b}} \mathrm{a}$

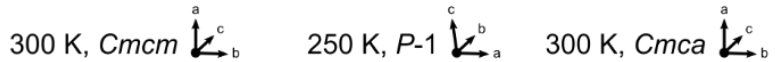

(d) Bond length distortion

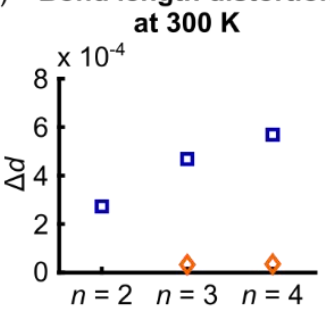

Bond angle variance at $300 \mathrm{~K}$

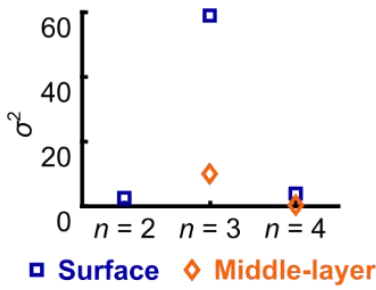

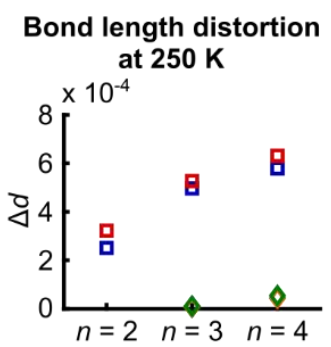

Bond angle variance

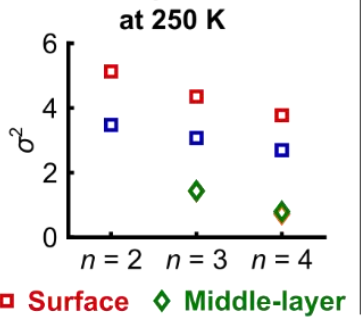

(e)

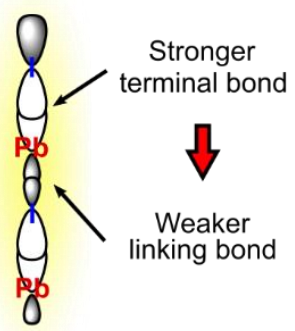

(f) Axial Pb-I Bond Length

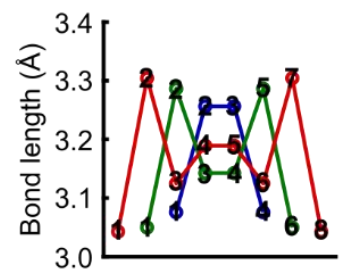

○ $n=2$ BA-MAPbI $\circ n=3$ BA-MAPbI $\circ n=4$ BA-MAPbI

(g) Axial Pb-( $\mu$-I)-Pb Angle

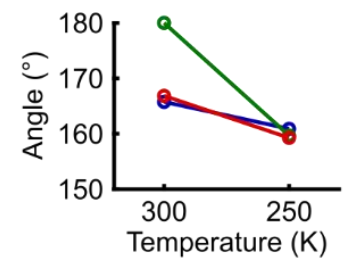

Equatorial $\mathrm{Pb}-(\mu-\mathrm{I})-\mathrm{Pb}$ Angle

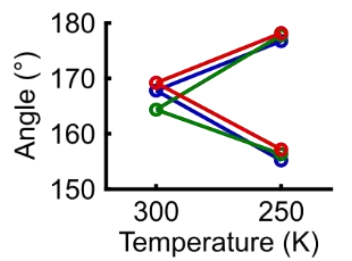

Figure 4. Analysis of crystal structures for $n=2$, 3, and 4 BA-MAPbI. (a) Structural phase transition of $n=2 \mathrm{BA}-\mathrm{MAPbI}$ from orthorhombic $\mathrm{Cmcm}$ at $300 \mathrm{~K}$ to triclinic $P-1$ at $250 \mathrm{~K}$. (b) Structural phase transition of $n=3 \mathrm{BA}-\mathrm{MAPbI}$ from orthorhombic $C m c a$ at $300 \mathrm{~K}$ to triclinic $P-1$ at $250 \mathrm{~K}$. (c) Structural phase transition of $n=4 \mathrm{BA}-\mathrm{MAPbI}$ from orthorhombic $\mathrm{Cmcm}$ at $300 \mathrm{~K}$ to triclinic $P-1$ at $250 \mathrm{~K}$. Structurally similar PbI6 octahedra are labelled by blue, orange, red, and green colors. (Disorder is omitted in all illustrations for clarity.) (d) Bond length distortion $(\Delta d$, top) and bond angle variance ( $\sigma^{2}$, bottom) of each type of PbI6 octahedra of $n=2,3$, and 4 BA$\mathrm{MAPbI}$ at $300 \mathrm{~K}$ (left) and $250 \mathrm{~K}$ (right). (e) Schematic diagram showing the orbital overlap of a terminal $\mathrm{Pb}-\mathrm{I}$ bond and a linking $\mathrm{Pb}-\mathrm{I}$ bond. (f) Plot of axial $\mathrm{Pb}-\mathrm{I}$ bond length starting from the top of the inorganic quantum wells of $n=2 \mathrm{BA}-\mathrm{MAPbI}$ (blue), $n=3 \mathrm{BA}-\mathrm{MAPbI}$ (green), and $n=4$ $\mathrm{BA}-\mathrm{MAPbI}$ (red). The number on the plot indicates the order of the bond starting from the top of the inorganic quantum wells. (g) Evolution of $\mathrm{Pb}-(\mu-\mathrm{I})-\mathrm{Pb}$ angle in axial (left) and equatorial (right) directions of $n=2 \mathrm{BA}-\mathrm{MAPbI}$ (blue), $n=3 \mathrm{BA}-\mathrm{MAPbI}$ (green), and $n=4 \mathrm{BA}-\mathrm{MAPbI}$ (red). 
When $n=2,3$, and $4 \mathrm{BA}-\mathrm{MAPbI}$ are cooled down to $250 \mathrm{~K}$, the $2 \mathrm{D}$ LHPs retain their staggered geometry but undergo phase transitions to a lower symmetry space group of triclinic $P$-1 (Figure 4a-c). As a result, both surface $\mathrm{PbI}_{6}$ octahedra and middle-layer PbI6 octahedra bifurcate into two families, each with different distortions yet similar magnitudes (Figure $4 \mathrm{~d}$ right panel). The phase transformation is accompanied by a significant change in the $\mathrm{Pb}-(\mu-\mathrm{I})-\mathrm{Pb}$ bond angles (Figure $4 \mathrm{~g})$ : (i) the $\mathrm{Pb}-(\mu-\mathrm{I})-\mathrm{Pb}$ bond angles in axial direction reduce to $\sim 160^{\circ}$, leading to significant increases in out-of-plane tilting across the three samples; and (ii) the $\mathrm{Pb}-(\mu-\mathrm{I})-\mathrm{Pb}$ bond angles in equatorial direction with initial values of $\sim 164-172^{\circ}$ separate into two groups with lower bond angles of $\sim 153-160^{\circ}$ and higher bond angles of $\sim 175-180^{\circ}$. This alteration in bond angles suggests a higher degree of distortion with respect to the ideal perovskite structure with all $\mathrm{Pb}-(\mu-\mathrm{I})-\mathrm{Pb}$ bond angles being $180^{\circ}$. In addition, the middle-layer $\mathrm{PbI}_{6}$ octahedra of $n=3 \mathrm{BA}-\mathrm{MAPbI}$ in this phase shows an out-of-plane tilting, unlike in the higher-temperature phase.

\section{- 5.2 Structural comparison of iodide 2D LHP crystals with different organic spacer lengths}

Previous reports on $n=1$ iodide 2D LHP have shown that the number of phase transitions and transition temperatures are dependent on the length of organic spacer. ${ }^{28-30}$ In this study, we observed that $n=2$ BA-MAPbI (BA = butylammonium), $n=2$ PA-MAPbI $(\mathrm{PA}=$ pentylammonium), and $n=2 \mathrm{HA}-\mathrm{MAPbI}(\mathrm{HA}=$ hexylammonium) exhibited one, two, and one phase transitions between 100-300 K, 100-363 K, and 100-385 K, respectively (Figures 4a, 5a, and 5b). The highest-temperature phases of these three samples all share the same orthorhombic Cmcm space group. As the temperature decreases, structural distortions lower the crystal symmetry for all three materials. When cooled, the crystal symmetry of $n=2 \mathrm{BA}-\mathrm{MAPbI}$ are lowered to 
triclinic $P-1$, and those of $n=2 \mathrm{PA}-\mathrm{MAPbI}$ and $\mathrm{HA}-\mathrm{MAPbI}$ are lowered to monoclinic $C 2 / c$, with an additional lowering of symmetry to $P 2{ }_{1} / m$ in the case of $n=2 \mathrm{PA}-\mathrm{MAPbI}$.

Table 2. Crystal data and structure refinement for $n=2 \mathrm{PA}-\mathrm{MAPbI}, n=2 \mathrm{HA}-\mathrm{MAPbI}$, and $n=2$ $\mathrm{BA}-\mathrm{FAPbI}$

\begin{tabular}{|c|c|c|c|c|c|c|c|}
\hline & \multicolumn{3}{|c|}{$n=2$ PA-MAPbI } & \multicolumn{2}{|c|}{$n=2 \mathrm{HA}-\mathrm{MAPbI}$} & \multicolumn{2}{|c|}{$n=2 \mathrm{BA}-\mathrm{FAPbI}$} \\
\hline $\begin{array}{l}\text { Chemical } \\
\text { formula }\end{array}$ & \multicolumn{3}{|c|}{$\mathrm{C}_{11} \mathrm{H}_{34} \mathrm{I}_{7} \mathrm{~N}_{3} \mathrm{~Pb}_{2}$} & \multicolumn{2}{|c|}{$\mathrm{C}_{13} \mathrm{H}_{38} \mathrm{I}_{7} \mathrm{~N}_{3} \mathrm{~Pb}_{2}$} & \multicolumn{2}{|c|}{$\mathrm{C}_{9} \mathrm{H}_{29} \mathrm{I}_{7} \mathrm{~N}_{4} \mathrm{~Pb}_{2}$} \\
\hline$M_{\mathrm{r}}$ & \multicolumn{3}{|c|}{1511.09} & \multicolumn{2}{|c|}{1539.14} & \multicolumn{2}{|c|}{1496.04} \\
\hline $\begin{array}{l}\text { Temperature } \\
\text { (K) }\end{array}$ & 363 & 330 & 250 & 385 & 300 & 300 & 230 \\
\hline Crystal system & Orthorhombic & Monoclinic & Monoclinic & Orthorhombic & Monoclinic & Orthorhombic & Triclinic \\
\hline Space group & $\mathrm{Cmcm}$ & $C 2 / c$ & $P 2_{1} / m$ & $\mathrm{Cmcm}$ & $C 2 / c$ & $\mathrm{Cmcm}$ & $P-1$ \\
\hline$a(\AA)$ & $42.539(10)$ & $41.588(14)$ & $8.8283(8)$, & $46.859(6)$ & $45.481(13)$ & $39.204(5)$ & $8.858(2)$ \\
\hline$b(\AA)$ & $8.993(2)$ & $9.000(3)$ & $41.044(4)$ & $8.9403(12)$ & $8.929(2)$ & $8.9490(12)$ & $9.137(2)$ \\
\hline$c(\AA)$ & $8.902(2)$ & $8.901(3)$ & $8.9886(8)$ & $8.9064(12)$ & $8.816(2)$ & $8.9431(11)$ & $\begin{array}{l}38.382 \\
(9)\end{array}$ \\
\hline$\alpha\left(^{\circ}\right)$ & 90 & 90 & 90 & 90 & 90 & 90 & $\begin{array}{l}90.140 \\
(3)\end{array}$ \\
\hline$\beta\left(^{\circ}\right)$ & 90 & $95.269(6)$ & \begin{tabular}{|l|}
90.8995 \\
$(14)$
\end{tabular} & 90 & $98.159(5)$ & 90 & \begin{tabular}{|l}
90.154 \\
$(4)$
\end{tabular} \\
\hline$\gamma\left({ }^{\circ}\right)$ & 90 & 90 & 90 & 90 & 90 & 90 & $\begin{array}{l}90.009 \\
(3)\end{array}$ \\
\hline$V\left(\AA^{3}\right)$ & $3405.5(13)$ & $3317.5(19)$ & $3256.6(5)$ & $3731.2(9)$ & $3544.0(17)$ & $3137.6(7)$ & $\begin{array}{l}3106.5 \\
(12) \\
\end{array}$ \\
\hline$Z$ & 4 & 4 & 4 & 4 & 4 & 4 & 4 \\
\hline$\mu\left(\mathrm{mm}^{-1}\right)$ & 16.22 & 16.65 & 16.97 & 14.81 & 15.59 & 17.61 & 17.78 \\
\hline $\begin{array}{l}\text { Crystal size } \\
(\mathrm{mm})\end{array}$ & $\begin{array}{l}0.14 \times 0.12 \times \\
0.06\end{array}$ & $\begin{array}{l}0.14 \times 0.12 \\
\times 0.06\end{array}$ & $\begin{array}{l}0.14 \times 0.12 \\
\times 0.06\end{array}$ & $\begin{array}{l}0.12 \times 0.10 \times \\
0.04\end{array}$ & $\begin{array}{l}0.12 \times 0.10 \\
\times 0.04\end{array}$ & $\begin{array}{l}0.12 \times 0.08 \times \\
0.04\end{array}$ & $\begin{array}{l}0.12 \times \\
0.08 \times \\
0.04\end{array}$ \\
\hline $\begin{array}{l}\text { No. of } \\
\text { measured } \\
\text { reflections }\end{array}$ & 29137 & 24756 & 56672 & 25310 & 27856 & 22442 & 24070 \\
\hline $\begin{array}{l}\text { No. of } \\
\text { independent } \\
\text { reflections }\end{array}$ & 1640 & 2949 & 5860 & 1794 & 3169 & 1526 & 10698 \\
\hline $\begin{array}{l}\text { No. of } \\
\text { observed }[I> \\
2 \sigma(I)] \\
\text { reflections }\end{array}$ & 1298 & 2347 & 4179 & 841 & 2702 & 1009 & 6276 \\
\hline$R_{\text {int }}$ & 0.029 & 0.053 & 0.048 & 0.052 & 0.061 & 0.067 & 0.051 \\
\hline
\end{tabular}




\begin{tabular}{|l|l|l|l|l|l|l|l|}
\hline $\begin{array}{l}(\sin \theta / \lambda)_{\max }\left(\AA^{-}\right. \\
1)\end{array}$ & 0.596 & 0.596 & 0.596 & 0.595 & 0.601 & 0.598 & 0.597 \\
\hline $\begin{array}{l}R\left[F^{2}>\right. \\
\left.2 \sigma\left(F^{2}\right)\right], \\
w R\left(F^{2}\right), S\end{array}$ & $\begin{array}{l}0.026,0.070, \\
1.08\end{array}$ & $\begin{array}{l}0.058, \\
0.158,1.11\end{array}$ & $\begin{array}{l}0.054, \\
0.143,1.07\end{array}$ & $\begin{array}{l}0.050,0.196, \\
1.05\end{array}$ & $\begin{array}{l}0.097, \\
0.265,1.08\end{array}$ & $\begin{array}{l}0.056,0.118, \\
1.06\end{array}$ & $\begin{array}{l}0.100, \\
0.269, \\
1.05\end{array}$ \\
\hline $\begin{array}{l}\text { No. of } \\
\text { reflections }\end{array}$ & 1640 & 2949 & 5860 & 1794 & 3169 & 1526 & 10698 \\
\hline $\begin{array}{l}\text { No. of } \\
\text { parameters }\end{array}$ & 99 & 134 & 262 & 119 & 133 & 103 & 443 \\
\hline $\begin{array}{l}\text { No. of } \\
\text { restraints }\end{array}$ & 48 & 49 & 124 & 72 & 69 & 61 & 283 \\
\hline $\begin{array}{l}\Delta \rho_{\max }, \Delta \rho_{\min } \\
\left(\mathrm{e} \AA^{-3}\right)\end{array}$ & $1.08,-0.72$ & $2.24,-2.66$ & $2.38,-1.80$ & $1.01,-0.92$ & $3.96,-2.62$ & $2.01,-1.64$ & $\begin{array}{l}3.43,- \\
4.31\end{array}$ \\
\hline
\end{tabular}

The structures of $n=2 \mathrm{BA}-\mathrm{MAPbI}, n=2 \mathrm{PA}-\mathrm{MAPbI}$, and $n=2 \mathrm{HA}-\mathrm{MAPbI}$ in orthorhombic Cmcm space group share many similarities. Firstly, in each structure, all PbI6 octahedra are equivalent. Adjacent octahedra have out-of-phase out-of-plane tilting, and larger tilting angles are observed in the $\mathrm{T}-\mathrm{Ax} \mathrm{Pb}-\mathrm{I}$ bonds compared to the $\mathrm{L}-\mathrm{Ax} \mathrm{Pb}-\mathrm{I}$ bonds. The latter reflects the larger space required to accommodate an organic spacer molecule compared to a MA cation. A closer inspection on these tilting angles show that there are decreasing trends in both angles as the organic spacer is increased (Figure 5d), and these changes are accompanied by increasing $\mathrm{Pb}-(\mu-\mathrm{I})-\mathrm{Pb}$ angles closer to $180^{\circ}$ (Figure 5e). An explanation for these behaviors can be given by noting that iodide 2D LHPs consist of interconnected inorganic slabs and organic spacer layers. In the organic spacer layer, there is an interaction between organic spacer molecules from different slabs, resulting in a stress in the layer. This stress can be transmitted to the inorganic slab through the carbon chain of the organic spacer molecule, resulting in the distortion of the $\mathrm{PbI}_{6}$ octahedra. As the organic spacer length increases, the stress is more absorbed by the longer carbon chain, and less is transmitted to the $\mathrm{PbI} 6$ octahedra. As a result, the PbI6 octahedra have a smaller degree of distortion in the sample with longer organic spacer length. 
(a)

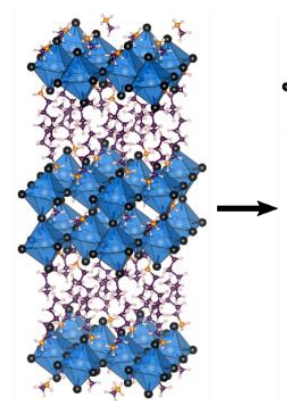

$363 \mathrm{~K}, \mathrm{Cmcm} \stackrel{\mathrm{a}}{\mathfrak{K}_{\mathrm{b}}^{\mathrm{b}}}$ $n=2$ PA-MAPbI

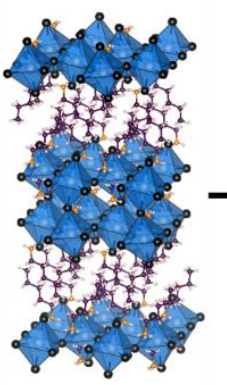

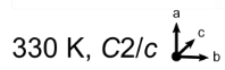

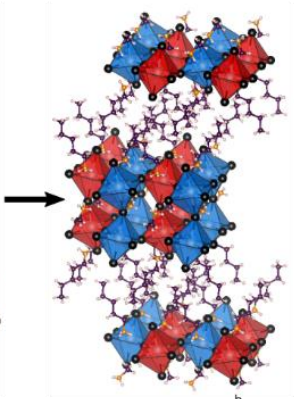

$250 \mathrm{~K}, P 2_{1} / m \stackrel{\mathrm{L}}{\mathrm{a}}$ 。 (c) $\quad n=2$ BA-FAPbl

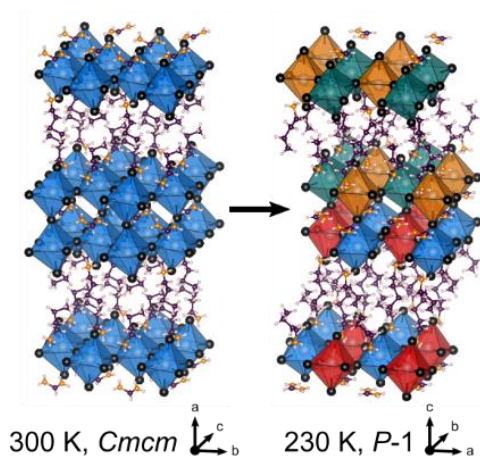

(d) Out-of-plane Tilting Angle

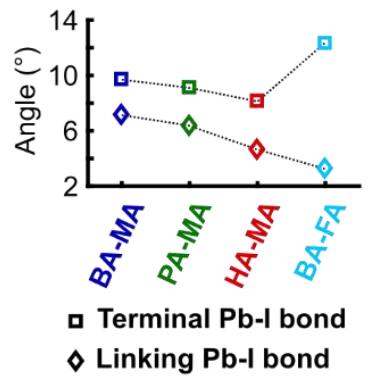

(b)

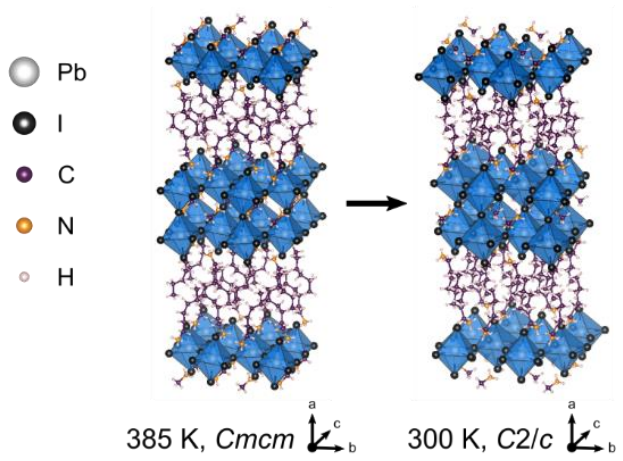

(e) Axial Pb-( $\mu$-I)-Pb Angle Equatorial $\mathrm{Pb}-(\mu-\mathrm{I})-\mathrm{Pb}$ Angle
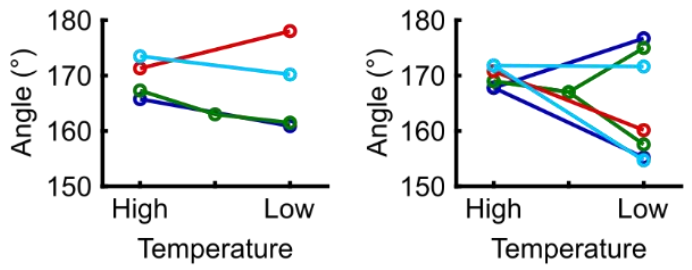

○ $n=2$ BA-MAPbI $\circ n=2$ HA-MAPbl

$\circ n=2$ PA-MAPbI $\circ n=2$ BA-FAPbI

Figure 5. Analysis of crystal structures for $n=2$ iodide $2 D$ LHP with changing organic spacer cation ( $L$ ) or A-site cation. (a) Structural phase transition of $n=2$ PA-MAPbI from orthorhombic $\mathrm{Cmcm}$ at $363 \mathrm{~K}$ to monoclinic $C 2 / c$ at $330 \mathrm{~K}$ and monoclinic $P 21 / m$ at $250 \mathrm{~K}$. (b) Structural phase transition of $n=2 \mathrm{HA}-\mathrm{MAPbI}$ from orthorhombic $C m c m$ at $385 \mathrm{~K}$ to monoclinic $C 2 / c$ at $300 \mathrm{~K}$. (c) Structural phase transition of $n=2 \mathrm{BA}-\mathrm{FAPbI}$ from orthorhombic $\mathrm{Cmcm}$ at $300 \mathrm{~K}$ to triclinic $P-1$ at $230 \mathrm{~K}$. (Disorder is omitted in all illustrations for clarity.) (d) Graph showing the out-ofplane tilting angle of terminal and linking $\mathrm{Pb}-\mathrm{I}$ bonds in $n=2 \mathrm{BA}-\mathrm{MAPbI}$ (blue), $n=2 \mathrm{PA}-\mathrm{MAPbI}$ (green), $n=2 \mathrm{HA}-\mathrm{MAPbI}$ (red), and $n=2 \mathrm{BA}-\mathrm{FAPbI}$ (cyan). (e) Evolution of $\mathrm{Pb}-(\mu-\mathrm{I})-\mathrm{Pb}$ angle in axial (left) and equatorial (right) directions of $n=2 \mathrm{BA}-\mathrm{MAPbI}$ (blue), $n=2 \mathrm{PA}-\mathrm{MAPbI}$ (green), $n=2 \mathrm{HA}-\mathrm{MAPbI}$ (red), and $n=2 \mathrm{BA}-\mathrm{FAPbI}$ (cyan).

Unlike $n=2 \mathrm{BA}-\mathrm{MAPbI}$ and $n=2 \mathrm{HA}-\mathrm{MAPbI}, n=2 \mathrm{PA}-\mathrm{MAPbI}$ exhibits two phase transitions over the temperature range investigated (Figure 6a). At $330 \mathrm{~K}, n=2$ PA-MAPbI adopts a monoclinic $C 2 / c$ structure with all $\mathrm{PbI}_{6}$ octahedra being equivalent. Compared to the highertemperature orthorhombic phase, the inorganic sublattice in the monoclinic phase maintains a similar structure with out-of-phase out-of-plane tilting, but with slightly lower $\mathrm{Pb}-(\mu-\mathrm{I})-\mathrm{Pb}$ bond 
angles (Figure 5e). However, a significant difference is observed in the organic spacer layer with an increase in the corrugation tilt (defined as the angle between the organic spacer chain and the normal vector of the inorganic slab) from $26.7^{\circ}$ to $40.6^{\circ}$. When the temperature is further reduced to $250 \mathrm{~K}$, the crystal symmetry is lowered to $P 21 / m$. In this phase, two types of PbI6 octahedra with different distortion are observed, similar to the $P-1$ phase of $n=2$ BA-MAPbI at $250 \mathrm{~K}$. Nevertheless, the two types of $\mathrm{PbI}_{6}$ octahedra in $n=2 \mathrm{PA}-\mathrm{MAPbI}$ only alternate along the lateral direction, unlike those in $n=2 \mathrm{BA}-\mathrm{MAPbI}$ which alternate in both vertical and lateral directions. In this phase, the $\mathrm{Pb}-(\mu-\mathrm{I})-\mathrm{Pb}$ bond angles in the axial direction decrease further, but the $\mathrm{Pb}-(\mu-\mathrm{I})-$ $\mathrm{Pb}$ bond angles in the equatorial direction are separated into two groups with lower bond angles of less than $160^{\circ}$ and higher bond angles of more than $173^{\circ}$, similar to the transformation discussed above in the case of $n=2,3$ and $4 \mathrm{BA}-\mathrm{MAPbI}$. Although significant transformation is observed in the inorganic sublattice, the change in the organic spacer layer is negligible with a small increase of corrugation tilt by less than $1^{\circ}$. As a result, the phase transition from the orthorhombic $\mathrm{Cmcm}$ phase to monoclinic $C 2 / c$ phase of $n=2 \mathrm{PA}-\mathrm{MAPbI}$ is primarily defined by a transition in the organic spacer layer, and the phase transition from the monoclinic $C 2 / c$ phase to the $P 2{ }_{1} / m$ phase is primarily defined by a transition in the inorganic sublattice.

For $n=2 \mathrm{HA}-\mathrm{MAPbI}$, only one transition - from the orthorhombic $\mathrm{Cmcm}$ to the monoclinic $C 2 / \mathrm{c}$ - is observed (Figure 5b). This transition is similar to that of $n=2$ PA-MAPbI from the orthorhombic $\mathrm{Cmcm}$ to the monoclinic $C 2 / c$ : equivalence of $\mathrm{PbI}_{6}$ octahedra is maintained, $\mathrm{Pb}-(\mu$ I)- $\mathrm{Pb}$ bond angles in the equatorial direction decrease, and the corrugation tilts of the organic spacers increase. However, unlike other iodide 2D LHPs discussed so far which have smaller Pb$(\mu-\mathrm{I})-\mathrm{Pb}$ bond angles in the axial direction at lower temperature, $n=2$ HA-MAPbI has a higher value of this bond angle of $178^{\circ}$, very close to the ideal geometry of $180^{\circ}$ (Figure 6e). In addition, 
there is a significant in-plane rotation of the $\mathrm{PbI}_{6}$ octahedra in the monoclinic $C 2 / c$ phase, unlike any other phases of $n=2$ iodide 2D LHPs discussed so far.

\section{- 5.3 Structural comparison of iodide 2D LHP crystals with different A-site cations}

Unlike $n=1$ iodide 2D LHPs, iodide 2D LHPs with $n=2$ and above include an A-site cation. In this study, we investigated the effect of replacing the A-site cation methylammonium (MA) for formamidinium (FA). At $300 \mathrm{~K}$, both $n=2 \mathrm{BA}-\mathrm{MAPbI}$ and $n=2 \mathrm{BA}-\mathrm{FAPbI}$ adopt the same orthorhombic $\mathrm{Cmcm}$ phase, and have similar appearance (Figures $4 \mathrm{a}$ and 5c). Nevertheless, all $\mathrm{Pb}$ $(\mu-\mathrm{I})-\mathrm{Pb}$ bond angles of $n=2 \mathrm{BA}-\mathrm{FAPbI}$ are significantly higher and closer to $180^{\circ}$ (Figure $5 \mathrm{e}$ ).

The out-of-plane tilting of the T-Ax and the L-Ax Pb-I bonds in $n=2 \mathrm{BA}-\mathrm{FAPbI}$ also increase and decrease to the values of $12^{\circ}$ and $3.3^{\circ}$, respectively (Figure $5 \mathrm{~d}$ ). These dramatic changes are unlikely to be the results of an increase in the cation size alone, and the statistically enhanced probability of forming hydrogen bonds by the increased number of FA protons compared to MA $(4 \text { versus } 3)^{60}$ must play an important role.

The low temperature phase of $n=2 \mathrm{BA}-\mathrm{FAPbI}$ is similar to that of $n=2 \mathrm{BA}-\mathrm{MAPbI}$. At $230 \mathrm{~K}, n$ $=2$ BA-FAPbI crystallizes in triclinic $P-1$ phase with lower symmetry compared to the orthorhombic $\mathrm{Cmcm}$ at $300 \mathrm{~K}$ (Figure 5c). In the triclinic $P-1$ phase, $n=2 \mathrm{BA}-\mathrm{FAPbI}$ has four types of $\mathrm{PbI}_{6}$ octahedra with different distortion. Although $\mathrm{Pb}-(\mu-\mathrm{I})-\mathrm{Pb}$ bond angles in the axial direction of $n=2 \mathrm{BA}-\mathrm{FAPbI}$ in triclinic $P-1$ phase are still higher than those of $n=2 \mathrm{BA}-\mathrm{MAPbI}$ with equivalent structure (Figure $5 \mathrm{e}$ ), the average $\mathrm{Pb}-(\mu-\mathrm{I})-\mathrm{Pb}$ bond angle in the equatorial direction of $n=2 \mathrm{BA}-\mathrm{FAPbI}$ is lower at $163.2^{\circ}$, compared to $165.7^{\circ}$ for $n=2 \mathrm{BA}-\mathrm{MAPbI}$. For the organic spacer layers, similar corrugation tilting within a few degrees is observed.

\section{- 5.4 Near-room-temperature structural transformations in the organic spacer layer}


Similarities were observed across all iodide 2D LHPs in their transformation from orthorhombic to lower-symmetry phases as the sample is cooled (Figure 6). In all samples, the high-temperature phase shows interdigitation of the organic cation spacers; the extent of interdigitation increases when sample is cooled below its phase transition temperature. Furthermore, all transitions (except the second transition of $n=2 \mathrm{PA}-\mathrm{MAPbI}$ between monoclinic $C 2 / c$ and monoclinic $P 2_{1} / m$ ) are accompanied by significant changes in the corrugation tilt angle of the organic spacer molecules. We characterized these transitions as freezing/melting transitions of the organic sublattice based on differential scanning calorimetry (DSC) measurements and thermodynamic calculation. These transformations will be described more fully in a forthcoming publication. 
(a)

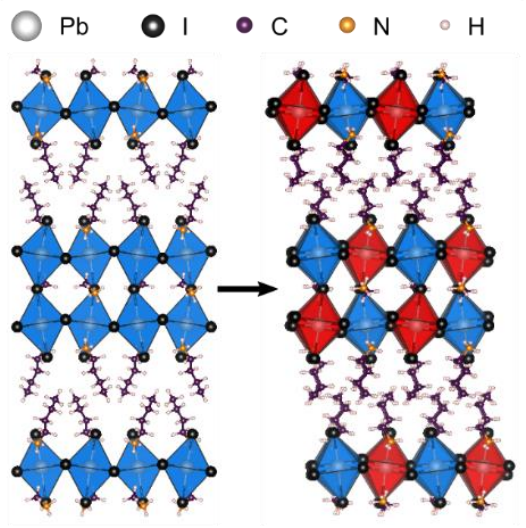

$300 \mathrm{~K}, \mathrm{Cmcm} \stackrel{\mathrm{a}}{\mathrm{L}} \mathrm{b} \quad 250 \mathrm{~K}, P-1 \stackrel{\mathrm{L}}{\longrightarrow} \mathrm{a}$ (b)

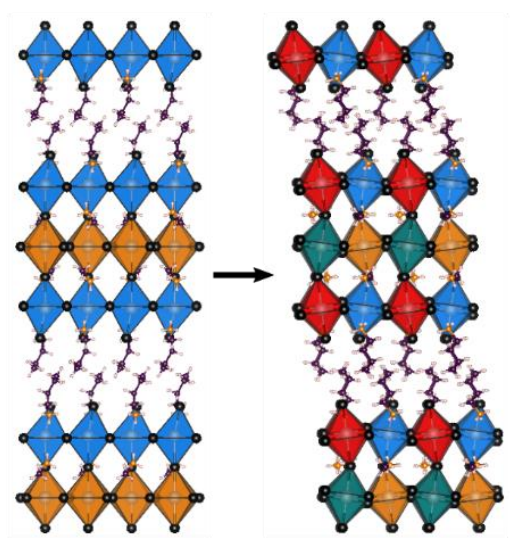

$300 \mathrm{~K}, \mathrm{Cmca} \stackrel{\mathrm{a}}{\longleftrightarrow} \mathrm{b}$

$250 \mathrm{~K}, P-1 \overbrace{\mathrm{a}}$ (c)

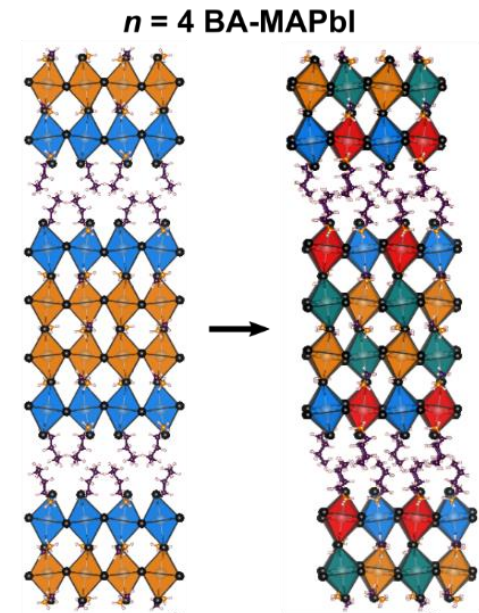

(d)

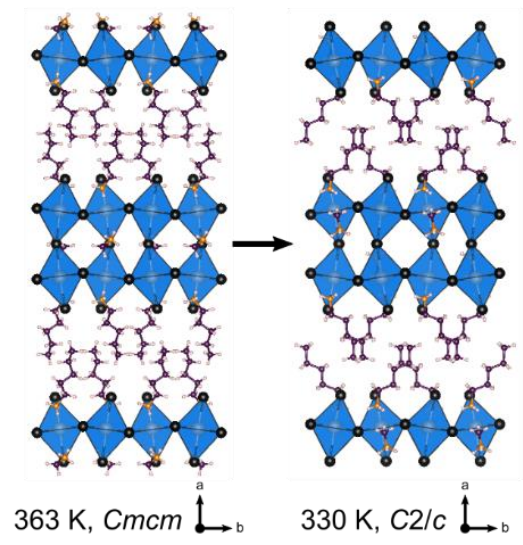

(e) $\quad n=2$ HA-MAPbI

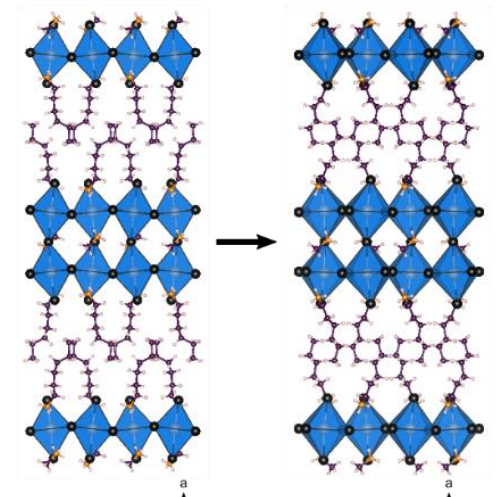

(f)

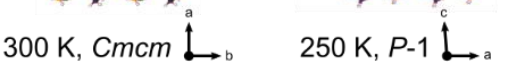

$n=2$ BA-FAPbI

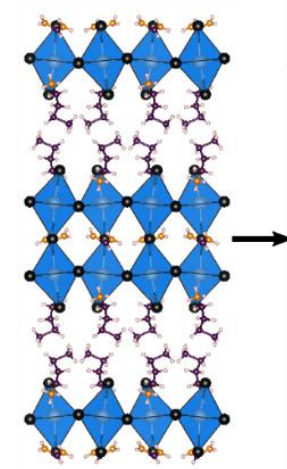

$300 \mathrm{~K}, \mathrm{Cmcm} \stackrel{\mathrm{a}}{\longrightarrow}$ 。

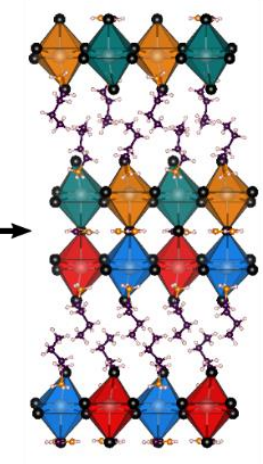

$230 \mathrm{~K}, P-1 \stackrel{\mathrm{L}}{\longrightarrow}$ a

Figure 6. Structural phase transitions viewed from the side to highlight the transitions of the organic spacer layers in (a) $n=2 \mathrm{BA}-\mathrm{MAPbI}$, (b) $n=3 \mathrm{BA}-\mathrm{MAPbI}$, (c) $n=4 \mathrm{BA}-\mathrm{MAPbI}$, (d) $n=$ 2 BA-FAPbI, (e) $n=2$ PA-MAPbI, and (f) $n=2$ HA-MAPbI. (Disorder is omitted in all illustrations for clarity.)

\section{Synthesis of bromide 2D LHP crystals}

The cooling-induced crystallization method we described for the synthesis of iodide 2D LHPs could be modified to prepare bromide 2D LHPs, as well. As explained in the experimental section, firstly, a large volume of lead (II) bromide $\left(\mathrm{PbBr}_{2}\right)$ solution was prepared by reacting $\mathrm{PbO}$ with hydrogen bromide $(\mathrm{HBr})$ solution under reflux. A small volume of this solution was then 
transferred into a small vial. Without the addition of $\mathrm{H}_{3} \mathrm{PO}_{2}$, a small volume of BA was added and white precipitate of $n=1 \mathrm{BA}-\mathrm{PbBr}$ formed. In a separate vial, a solution of the A-site cation bromide salt was prepared by dissolving the salt in $\mathrm{HBr}$. This solution was subsequently added to the solution containing white precipitate to form a combined solution. This combined solution was heated until clear, then slowly cooled to produce crystals of bromide 2D LHPs. Finally, these crystals were collected by suction filtration and dried under reduced pressure. Examples of crystals obtained by this method are shown in Figure $7 \mathrm{a}$.

When synthesizing bromide 2D LHPs, we found that the effects of each synthesis parameter on the purity of the product described earlier for iodide 2D LHPs also applied to the case of bromide 2D LHPs. However, bromide 2D LHPs exhibited faster precipitation rates, which resulted in the formation of smaller crystals. To grow larger bromide 2D LHPs, it was necessary to lower the concentration of each reagent by adding additional volume of $\mathrm{HBr}$ to the final solution.

Occasionally, we observed inhomogeneous orange colorings on bromide 2D LHPs during suction filtration and drying (Figure S10). These orange colorings were easily noticed in the cases of white $n=1 \mathrm{BA}-\mathrm{PbBr}$ and yellow $n=2 \mathrm{BA}-\mathrm{MAPbBr}$. Further investigation showed that the PL spectra obtained from orange regions was identical to the clear regions. Since no MA cation was involved in the synthesis of $n=1 \mathrm{BA}-\mathrm{PbBr}$, the orange coloring cannot be due to higher members of bromide 2D LHPs. One possible cause is the presence of molecular $\mathrm{Br}_{2}$ intercalated in the organic spacer layer, similarly to the previously reported intercalation of $\mathrm{I}_{2}$ in $2 \mathrm{D}$ LHPs. ${ }^{68}$

Figure 7(b) shows the Abs and the PL spectra of exfoliated $n=1,2$, and 3 bromide 2D LHPs with BA organic spacer. Only the absorption of the 1s excitonic resonance was captured in these experiments due to poor signal-to-noise ratio at wavelengths shorter than $\sim 420 \mathrm{~nm}$ in our 
measurements. The presence of a single PL peak corresponding to the measured absorption resonance confirmed the high phase-purity of these 2D LHP crystals.

(a)
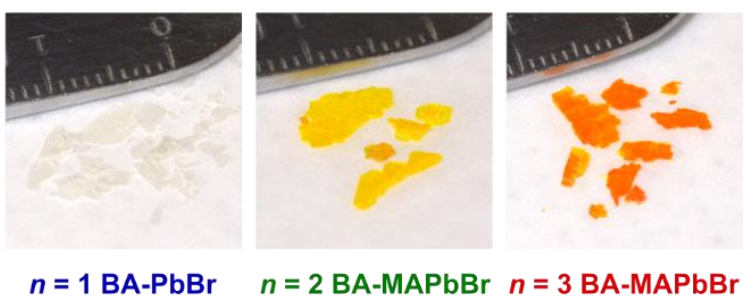

(b)
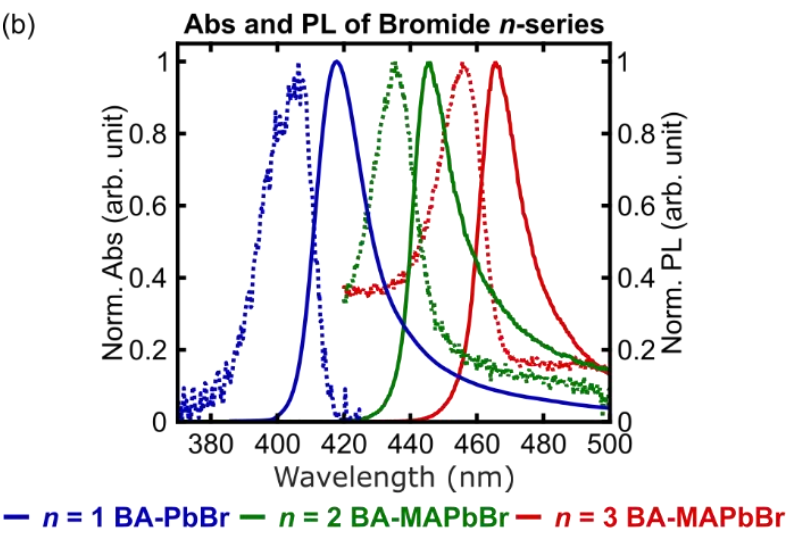

(c)

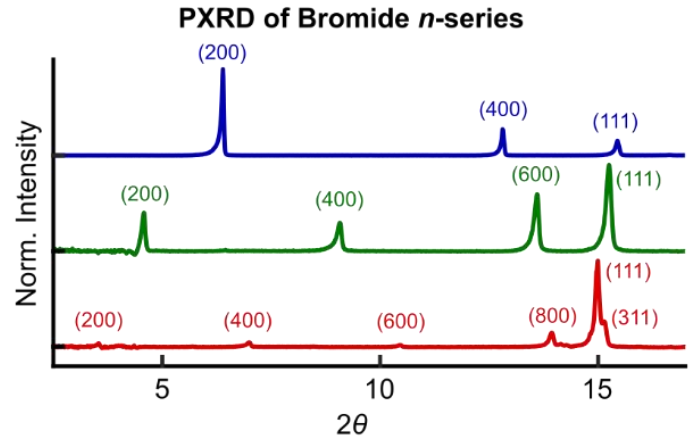

$-n=1 \mathrm{BA}-\mathrm{PbBr}-n=2 \mathrm{BA}-\mathrm{MAPbBr}-n=3 \mathrm{BA}-\mathrm{MAPbBr}$

Figure 7. Bromide 2D LHPs. (a) Photographs of 2D LHP crystals: $n=1 \mathrm{BA}-\mathrm{PbBr}$ (left), $n=2$ $\mathrm{BA}-\mathrm{MAPbBr}$ (middle), and $n=3 \mathrm{BA}-\mathrm{MAPbBr}$ (right). The spacing between minor ticks on the ruler is $1 \mathrm{~mm}$. (b) Absorption (dotted) and photoluminescence (solid) spectra of $n=1 \mathrm{BA}-\mathrm{PbBr}$ (blue), $n=2 \mathrm{BA}-\mathrm{MAPbBr}$ (green), and $n=3 \mathrm{BA}-\mathrm{MAPbBr}$ (red). (c) Powder X-ray diffractograms of $n=1 \mathrm{BA}-\mathrm{PbBr}$ (blue), $n=2 \mathrm{BA}-\mathrm{MAPbBr}$ (green), and $n=3 \mathrm{BA}-\mathrm{MAPbBr}$ (red). All peaks are labelled with their corresponding diffraction planes by analogy to the iodide series. 
The PXRD patterns of bromide 2D LHPs (Figure 7c) show the same features as those observed in their iodide counterparts. ${ }^{20}$ Due to the smaller size of bromine compared to iodine, the inorganic slabs of bromide 2D LHPs are thinner, and all peaks shift to higher $2 \theta$. The (111) peaks appear at $\sim 15^{\circ} 2 \theta$, while the number of evenly spaced (h00) peaks below the (111) peak is still $n+1$. The calculated stacking periods corresponding to these Bragg peaks for $n=1 \mathrm{BA}-\mathrm{PbBr}, n=2 \mathrm{BA}$ $\mathrm{MAPbBr}$, and $n=3 \mathrm{BA}-\mathrm{MAPbBr}$ are $1.38 \mathrm{~nm}, 1.95 \mathrm{~nm}$, and $2.52 \mathrm{~nm}$, respectively.

\section{Conclusions}

We have reported cooling-induced crystallization of high-quality 2D LHP crystals of $n=2$ BAMAPbI, $n=3$ BA-MAPbI, $n=4$ BA-MAPbI, $n=2$ PA-MAPbI, $n=2 \mathrm{HA}-\mathrm{MAPbI}$, and $n=2 \mathrm{BA}-$ FAPbI, as well as crystal structures for these compounds at multiple temperatures. The synthetic approach is generalizable to the bromide 2D LHP system, and high-quality crystals of $n=1$ BA$\mathrm{PbBr}, n=2 \mathrm{BA}-\mathrm{MAPbBr}$, and $n=3 \mathrm{BA}-\mathrm{MAPbBr}$ were demonstrated. Overall, this work illuminates broader structural trends in 2D alkylammonium LHPs, and the structural insights will provide a foundation for understanding thermal, electrical, and optical properties of 2D LHPs.

\section{Acknowledgements}

The authors thank Charles Settens for assistance with visualization of crystallographic data, Alexia Stollman for assistant with powder X-ray crystallography, Blair Anaman-Williams for assistance with the synthesis of bromide 2D LHPs, and the Ling lab in the Chemistry Department at Boston University for assistance with absorption measurements. This work was supported by the U.S. Department of Energy, Office of Science, Office of Basic Energy Sciences, under award no. DESC0019345. Powder XRD measurements were performed at the MRSEC Shared Experimental 
Facilities at MIT, supported by the National Science Foundation under award number DMR-0819762.

\section{Associated Content}

\section{Supporting Information}

The Supporting Information is available free of charge on the ACS Publications website at DOI: xxx. CCDC 1880158-1880170 contain the supplementary crystallographic data for this paper. These data are provided free of charge by The Cambridge Crystallographic Data Center.

Synthesis conditions, synthesis observation, bright-field micrographs of exfoliated flakes, optical characterization, and structural illustrations (PDF)

Single crystal X-ray crystallographic data for $n=2$ BA-MAPbI at $300 \mathrm{~K}$ (CIF)

Single crystal X-ray crystallographic data for $n=2$ BA-MAPbI at $250 \mathrm{~K}$ (CIF)

Single crystal X-ray crystallographic data for $n=3$ BA-MAPbI at $300 \mathrm{~K}$ (CIF)

Single crystal X-ray crystallographic data for $n=3$ BA-MAPbI at $250 \mathrm{~K}$ (CIF)

Single crystal X-ray crystallographic data for $n=4$ BA-MAPbI at $300 \mathrm{~K}$ (CIF)

Single crystal X-ray crystallographic data for $n=4$ BA-MAPbI at $250 \mathrm{~K}$ (CIF)

Single crystal X-ray crystallographic data for $n=2$ PA-MAPbI at $363 \mathrm{~K}(\mathrm{CIF})$

Single crystal X-ray crystallographic data for $n=2 \mathrm{PA}-\mathrm{MAPbI}$ at $330 \mathrm{~K}$ (CIF)

Single crystal X-ray crystallographic data for $n=2$ PA-MAPbI at $250 \mathrm{~K}$ (CIF) 
Single crystal X-ray crystallographic data for $n=2 \mathrm{HA}-\mathrm{MAPbI}$ at $385 \mathrm{~K}$ (CIF)

Single crystal X-ray crystallographic data for $n=2 \mathrm{HA}-\mathrm{MAPbI}$ at $300 \mathrm{~K}$ (CIF)

Single crystal X-ray crystallographic data for $n=2 \mathrm{BA}-\mathrm{FAPbI}$ at $300 \mathrm{~K}$ (CIF)

Single crystal X-ray crystallographic data for $n=2 \mathrm{BA}-\mathrm{FAPbI}$ at $230 \mathrm{~K}$ (CIF) 


\section{References}

(1) Smith, I. C.; Hoke, E. T.; Solis-Ibarra, D.; McGehee, M. D.; Karunadasa, H. I. A Layered Hybrid Perovskite Solar-Cell Absorber with Enhanced Moisture Stability. Angew. Chemie Int. Ed. 2014, 53 (42), 11232-11235. https://doi.org/10.1002/anie.201406466.

(2) Tsai, H.; Nie, W.; Blancon, J.-C.; Stoumpos, C. C.; Asadpour, R.; Harutyunyan, B.; Neukirch, A. J.; Verduzco, R.; Crochet, J. J.; Tretiak, S.; et al. High-Efficiency TwoDimensional Ruddlesden-Popper Perovskite Solar Cells. Nature 2016, 536 (7616), 312 316. https://doi.org/10.1038/nature18306.

(3) Mao, L.; Stoumpos, C. C.; Kanatzidis, M. G. Two-Dimensional Hybrid Halide Perovskites: Principles and Promises. J. Am. Chem. Soc. 2019, 141 (3), 1171-1190. https://doi.org/10.1021/jacs.8b10851.

(4) Cheng, Z.; Lin, J. Layered Organic-inorganic Hybrid Perovskites: Structure, Optical Properties, Film Preparation, Patterning and Templating Engineering. CrystEngComm 2010, 12 (10), 2646. https://doi.org/10.1039/c001929a.

(5) Weidman, M. C.; Seitz, M.; Stranks, S. D.; Tisdale, W. A. Highly Tunable Colloidal Perovskite Nanoplatelets through Variable Cation, Metal, and Halide Composition. ACS Nano 2016, 10 (8), 7830-7839. https://doi.org/10.1021/acsnano.6b03496.

(6) Blancon, J.-C.; Stier, A. V.; Tsai, H.; Nie, W.; Stoumpos, C. C.; Traoré, B.; Pedesseau, L.; Kepenekian, M.; Katsutani, F.; Noe, G. T.; et al. Scaling Law for Excitons in 2D Perovskite Quantum Wells. Nat. Commun. 2018, 9 (1), 2254. https://doi.org/10.1038/s41467-018-04659-X.

(7) Sichert, J. A.; Tong, Y.; Mutz, N.; Vollmer, M.; Fischer, S.; Milowska, K. Z.; García Cortadella, R.; Nickel, B.; Cardenas-Daw, C.; Stolarczyk, J. K.; et al. Quantum Size Effect in Organometal Halide Perovskite Nanoplatelets. Nano Lett. 2015, 15 (10), 65216527. https://doi.org/10.1021/acs.nanolett.5b02985.

(8) Zhou, N.; Shen, Y.; Li, L.; Tan, S.; Liu, N.; Zheng, G.; Chen, Q.; Zhou, H. Exploration of Crystallization Kinetics in Quasi Two-Dimensional Perovskite and High Performance Solar Cells. J. Am. Chem. Soc. 2018, 140 (1), 459-465. https://doi.org/10.1021/jacs.7b11157.

(9) Proppe, A. H.; Quintero-Bermudez, R.; Tan, H.; Voznyy, O.; Kelley, S. O.; Sargent, E. H. Synthetic Control over Quantum Well Width Distribution and Carrier Migration in LowDimensional Perovskite Photovoltaics. J. Am. Chem. Soc. 2018, 140 (8), 2890-2896. https://doi.org/10.1021/jacs.7b12551.

(10) Mao, L.; Ke, W.; Pedesseau, L.; Wu, Y.; Katan, C.; Even, J.; Wasielewski, M. R.; Stoumpos, C. C.; Kanatzidis, M. G. Hybrid Dion-Jacobson 2D Lead Iodide Perovskites. J. Am. Chem. Soc. 2018, 140 (10), 3775-3783. https://doi.org/10.1021/jacs.8b00542. 
(11) Mao, L.; Tsai, H.; Nie, W.; Ma, L.; Im, J.; Stoumpos, C. C.; Malliakas, C. D.; Hao, F.; Wasielewski, M. R.; Mohite, A. D.; et al. Role of Organic Counterion in Lead- and TinBased Two-Dimensional Semiconducting Iodide Perovskites and Application in Planar Solar Cells. Chem. Mater. 2016, 28 (21), 7781-7792. https://doi.org/10.1021/acs.chemmater.6b03054.

(12) Cao, D. H.; Stoumpos, C. C.; Farha, O. K.; Hupp, J. T.; Kanatzidis, M. G. 2D Homologous Perovskites as Light-Absorbing Materials for Solar Cell Applications. J. Am. Chem. Soc. 2015, 137 (24), 7843-7850. https://doi.org/10.1021/jacs.5b03796.

(13) Quan, L. N.; Zhao, Y.; García de Arquer, F. P.; Sabatini, R.; Walters, G.; Voznyy, O.; Comin, R.; Li, Y.; Fan, J. Z.; Tan, H.; et al. Tailoring the Energy Landscape in Quasi-2D Halide Perovskites Enables Efficient Green-Light Emission. Nano Lett. 2017, 17 (6), 3701-3709. https://doi.org/10.1021/acs.nanolett.7b00976.

(14) Tsai, H.; Nie, W.; Blancon, J.-C.; Stoumpos, C. C.; Soe, C. M. M.; Yoo, J.; Crochet, J.; Tretiak, S.; Even, J.; Sadhanala, A.; et al. Stable Light-Emitting Diodes Using Phase-Pure Ruddlesden-Popper Layered Perovskites. Adv. Mater. 2018, 30 (6), 1704217. https://doi.org/10.1002/adma.201704217.

(15) Wang, N.; Cheng, L.; Ge, R.; Zhang, S.; Miao, Y.; Zou, W.; Yi, C.; Sun, Y.; Cao, Y.; Yang, R.; et al. Perovskite Light-Emitting Diodes Based on Solution-Processed SelfOrganized Multiple Quantum Wells. Nat. Photonics 2016, 10 (11), 699-704. https://doi.org/10.1038/nphoton.2016.185.

(16) Congreve, D. N.; Weidman, M. C.; Seitz, M.; Paritmongkol, W.; Dahod, N. S.; Tisdale, W. A. Tunable Light-Emitting Diodes Utilizing Quantum-Confined Layered Perovskite Emitters. ACS Photonics 2017, 4 (3), 476-481. https://doi.org/10.1021/acsphotonics.6b00963.

(17) Han, S.; Wang, P.; Zhang, J.; Liu, X.; Sun, Z.; Huang, X.; Li, L.; Ji, C.; Zhang, W.; Teng, B.; et al. Exploring a Polar Two-Dimensional Multi-Layered Hybrid Perovskite of $\left(\mathrm{C}_{5} \mathrm{H}_{11} \mathrm{NH}_{3}\right)_{2}\left(\mathrm{CH}_{3} \mathrm{NH}_{3}\right) \mathrm{Pb}_{2} \mathrm{I}_{7}$ for Ultrafast-Responding Photodetection. Laser Photon. Rev. 2018, 12 (8), 1800060. https://doi.org/10.1002/lpor.201800060.

(18) Peng, W.; Yin, J.; Ho, K.-T.; Ouellette, O.; De Bastiani, M.; Murali, B.; El Tall, O.; Shen, C.; Miao, X.-H.; Pan, J.; et al. Ultralow Self-Doping in Two-Dimensional Hybrid Perovskite Single Crystals. Nano Lett. 2017, 17 (8), 4759-4767. https://doi.org/10.1021/acs.nanolett.7b01475.

(19) Sun, C.; Xu, G.; Jiang, X.-M.; Wang, G.-E.; Guo, P.-Y.; Wang, M.-S.; Guo, G.-C. Design Strategy for Improving Optical and Electrical Properties and Stability of Lead-Halide Semiconductors. J. Am. Chem. Soc. 2018, 140 (8), 2805-2811. https://doi.org/10.1021/jacs.7b10101.

(20) Li, L.; Sun, Z.; Wang, P.; Hu, W.; Wang, S.; Ji, C.; Hong, M.; Luo, J. Tailored Engineering of an Unusual $\left(\mathrm{C}_{4} \mathrm{H}_{9} \mathrm{NH}_{3}\right)_{2}\left(\mathrm{CH}_{3} \mathrm{NH}_{3}\right)_{2} \mathrm{~Pb}_{3} \mathrm{Br}_{10}$ Two-Dimensional Multilayered Perovskite Ferroelectric for a High-Performance Photodetector. Angew. Chemie Int. Ed. 
2017, 56 (40), 12150-12154. https://doi.org/10.1002/anie.201705836.

(21) Saparov, B.; Mitzi, D. B. Organic-Inorganic Perovskites: Structural Versatility for Functional Materials Design. Chem. Rev. 2016, 116 (7), 4558-4596. https://doi.org/10.1021/acs.chemrev.5b00715.

(22) Yin, J.; Maity, P.; Xu, L.; El-Zohry, A. M.; Li, H.; Bakr, O. M.; Brédas, J.-L.; Mohammed, O. F. Layer-Dependent Rashba Band Splitting in 2D Hybrid Perovskites. Chem. Mater. 2018, 30 (23), 8538-8545. https://doi.org/10.1021/acs.chemmater.8b03436.

(23) Chen, X.; Lu, H.; Li, Z.; Zhai, Y.; Ndione, P. F.; Berry, J. J.; Zhu, K.; Yang, Y.; Beard, M. C. Impact of Layer Thickness on the Charge Carrier and Spin Coherence Lifetime in TwoDimensional Layered Perovskite Single Crystals. ACS Energy Lett. 2018, 3 (9), 22732279. https://doi.org/10.1021/acsenergylett.8b01315.

(24) Smith, M. D.; Connor, B. A.; Karunadasa, H. I. Tuning the Luminescence of Layered Halide Perovskites. Chem. Rev. 2019, 119 (5), 3104-3139. https://doi.org/10.1021/acs.chemrev.8b00477.

(25) Whitfield, P. S.; Herron, N.; Guise, W. E.; Page, K.; Cheng, Y. Q.; Milas, I.; Crawford, M. K. Structures, Phase Transitions and Tricritical Behavior of the Hybrid Perovskite Methyl Ammonium Lead Iodide. Sci. Rep. 2016, 6 (1), 35685. https://doi.org/10.1038/srep35685.

(26) Harwell, J. R.; Payne, J. L.; Sajjad, M. T.; Heutz, F. J. L.; Dawson, D. M.; Whitfield, P. S.; Irvine, J. T. S.; Samuel, I. D. W.; Carpenter, M. A. Role of Lattice Distortion and A Site Cation in the Phase Transitions of Methylammonium Lead Halide Perovskites. Phys. Rev. Mater. 2018, 2 (6), 065404. https://doi.org/10.1103/PhysRevMaterials.2.065404.

(27) Stoumpos, C. C.; Malliakas, C. D.; Kanatzidis, M. G. Semiconducting Tin and Lead Iodide Perovskites with Organic Cations: Phase Transitions, High Mobilities, and NearInfrared Photoluminescent Properties. Inorg. Chem. 2013, 52 (15), 9019-9038. https://doi.org/10.1021/ic401215x.

(28) Billing, D. G.; Lemmerer, A. Synthesis, Characterization and Phase Transitions in the Inorganic-organic Layered Perovskite-Type Hybrids $\left[\left(\mathrm{C}_{n} \mathrm{H}_{2 n+1} \mathrm{NH}_{3}\right)_{2} \mathrm{PbI} 4\right], n=4,5$ and 6. Acta Crystallogr. Sect. B Struct. Sci. 2007, 63 (5), 735-747. https://doi.org/10.1107/S0108768107031758.

(29) Lemmerer, A.; Billing, D. G. Synthesis, Characterization and Phase Transitions of the Inorganic-organic Layered Perovskite-Type Hybrids $\left[\left(\mathrm{C}_{n} \mathrm{H}_{2 n+1} \mathrm{NH}_{3}\right)_{2} \mathrm{PbI}_{4}\right], n=7,8,9$ and 10. Dalt. Trans. 2012, 41 (4), 1146-1157. https://doi.org/10.1039/C0DT01805H.

(30) Billing, D. G.; Lemmerer, A. Synthesis, Characterization and Phase Transitions of the Inorganic-organic Layered Perovskite-Type Hybrids $\left[\left(\mathrm{C}_{n} \mathrm{H}_{2 n+1} \mathrm{NH}_{3}\right)_{2} \mathrm{PbI} 4\right](n=12,14,16$ and 18). New J. Chem. 2008, 32 (10), 1736. https://doi.org/10.1039/b805417g.

(31) Weidman, M. C.; Goodman, A. J.; Tisdale, W. A. Colloidal Halide Perovskite 
Nanoplatelets: An Exciting New Class of Semiconductor Nanomaterials. Chem. Mater. 2017, 29 (12), 5019-5030. https://doi.org/10.1021/acs.chemmater.7b01384.

(32) Shi, E.; Gao, Y.; Finkenauer, B. P.; Akriti, A.; Coffey, A. H.; Dou, L. Two-Dimensional Halide Perovskite Nanomaterials and Heterostructures. Chem. Soc. Rev. 2018, 47 (16), 6046-6072. https://doi.org/10.1039/C7CS00886D.

(33) Jagielski, J.; Kumar, S.; Yu, W.-Y.; Shih, C.-J. Layer-Controlled Two-Dimensional Perovskites: Synthesis and Optoelectronics. J. Mater. Chem. C 2017, 5 (23), 5610-5627. https://doi.org/10.1039/C7TC00538E.

(34) Dou, L. Emerging Two-Dimensional Halide Perovskite Nanomaterials. J. Mater. Chem. C 2017, 5 (43), 11165-11173. https://doi.org/10.1039/C7TC02863F.

(35) Huang, H.; Polavarapu, L.; Sichert, J. A.; Susha, A. S.; Urban, A. S.; Rogach, A. L. Colloidal Lead Halide Perovskite Nanocrystals: Synthesis, Optical Properties and Applications. NPG Asia Mater. 2016, 8 (11), e328. https://doi.org/10.1038/am.2016.167.

(36) Sun, S.; Yuan, D.; Xu, Y.; Wang, A.; Deng, Z. Ligand-Mediated Synthesis of ShapeControlled Cesium Lead Halide Perovskite Nanocrystals via Reprecipitation Process at Room Temperature. ACS Nano 2016, 10 (3), 3648-3657. https://doi.org/10.1021/acsnano.5b08193.

(37) Bekenstein, Y.; Koscher, B. A.; Eaton, S. W.; Yang, P.; Alivisatos, A. P. Highly Luminescent Colloidal Nanoplates of Perovskite Cesium Lead Halide and Their Oriented Assemblies. J. Am. Chem. Soc. 2015, 137 (51), 16008-16011. https://doi.org/10.1021/jacs.5b11199.

(38) Shamsi, J.; Dang, Z.; Bianchini, P.; Canale, C.; Stasio, F. Di; Brescia, R.; Prato, M.; Manna, L. Colloidal Synthesis of Quantum Confined Single Crystal CsPbBr 3 Nanosheets with Lateral Size Control up to the Micrometer Range. J. Am. Chem. Soc. 2016, 138 (23), 7240-7243. https://doi.org/10.1021/jacs.6b03166.

(39) Protesescu, L.; Yakunin, S.; Bodnarchuk, M. I.; Krieg, F.; Caputo, R.; Hendon, C. H.; Yang, R. X.; Walsh, A.; Kovalenko, M. V. Nanocrystals of Cesium Lead Halide Perovskites $\left(\mathrm{CsPbX}_{3}, \mathrm{X}=\mathrm{Cl}, \mathrm{Br}\right.$, and I): Novel Optoelectronic Materials Showing Bright Emission with Wide Color Gamut. Nano Lett. 2015, 15 (6), 3692-3696. https://doi.org/10.1021/nl5048779.

(40) Dou, L.; Wong, A. B.; Yu, Y.; Lai, M.; Kornienko, N.; Eaton, S. W.; Fu, A.; Bischak, C. G.; Ma, J.; Ding, T.; et al. Atomically Thin Two-Dimensional Organic-Inorganic Hybrid Perovskites. Science. 2015, 349 (6255), 1518-1521. https://doi.org/10.1126/science.aac7660.

(41) Liu, J.; Xue, Y.; Wang, Z.; Xu, Z.-Q.; Zheng, C.; Weber, B.; Song, J.; Wang, Y.; Lu, Y.; Zhang, Y.; et al. Two-Dimensional $\mathrm{CH}_{3} \mathrm{NH}_{3} \mathrm{PbI}_{3}$ Perovskite: Synthesis and Optoelectronic Application. ACS Nano 2016, 10 (3), 3536-3542. https://doi.org/10.1021/acsnano.5b07791. 
(42) Niu, L.; Liu, X.; Cong, C.; Wu, C.; Wu, D.; Chang, T. R.; Wang, H.; Zeng, Q.; Zhou, J.; Wang, X.; et al. Controlled Synthesis of Organic/Inorganic van Der Waals Solid for Tunable Light-Matter Interactions. Adv. Mater. 2015, 27 (47), 7800-7808. https://doi.org/10.1002/adma.201503367.

(43) Saidaminov, M. I.; Abdelhady, A. L.; Murali, B.; Alarousu, E.; Burlakov, V. M.; Peng, W.; Dursun, I.; Wang, L.; He, Y.; Maculan, G.; et al. High-Quality Bulk Hybrid Perovskite Single Crystals within Minutes by Inverse Temperature Crystallization. Nat. Commun. 2015, 6 (May), 7586. https://doi.org/10.1038/ncomms8586.

(44) Raghavan, C. M.; Chen, T.-P.; Li, S.-S.; Chen, W.-L.; Lo, C.-Y.; Liao, Y.-M.; Haider, G.; Lin, C.-C.; Chen, C.-C.; Sankar, R.; et al. Low-Threshold Lasing from 2D Homologous Organic-Inorganic Hybrid Ruddlesden-Popper Perovskite Single Crystals. Nano Lett. 2018, 18 (5), 3221-3228. https://doi.org/10.1021/acs.nanolett.8b00990.

(45) Shi, D.; Adinolfi, V.; Comin, R.; Yuan, M.; Alarousu, E.; Buin, A.; Chen, Y.; Hoogland, S.; Rothenberger, A.; Katsiev, K.; et al. Low Trap-State Density and Long Carrier Diffusion in Organolead Trihalide Perovskite Single Crystals. Science. 2015, 347 (6221), 519-522. https://doi.org/10.1126/science.aaa2725.

(46) Gong, X.; Voznyy, O.; Jain, A.; Liu, W.; Sabatini, R.; Piontkowski, Z.; Walters, G.; Bappi, G.; Nokhrin, S.; Bushuyev, O.; et al. Electron-phonon Interaction in Efficient Perovskite Blue Emitters. Nat. Mater. 2018, 17 (6), 550-556. https://doi.org/10.1038/s41563-018-0081-x.

(47) Stoumpos, C. C.; Cao, D. H.; Clark, D. J.; Young, J.; Rondinelli, J. M.; Jang, J. I.; Hupp, J. T.; Kanatzidis, M. G. Ruddlesden-Popper Hybrid Lead Iodide Perovskite 2D Homologous Semiconductors. Chem. Mater. 2016, 28 (8), 2852-2867. https://doi.org/10.1021/acs.chemmater.6b00847.

(48) Stoumpos, C. C.; Soe, C. M. M.; Tsai, H.; Nie, W.; Blancon, J.-C.; Cao, D. H.; Liu, F.; Traoré, B.; Katan, C.; Even, J.; et al. High Members of the 2D Ruddlesden-Popper Halide Perovskites: Synthesis, Optical Properties, and Solar Cells of $\left(\mathrm{CH}_{3}\left(\mathrm{CH}_{2}\right)_{3} \mathrm{NH}_{3}\right)_{2}\left(\mathrm{CH}_{3} \mathrm{NH}_{3}\right)_{4} \mathrm{~Pb}_{5} \mathrm{I}_{16}$. Chem 2017, 2 (3), 427-440. https://doi.org/10.1016/j.chempr.2017.02.004.

(49) Mitzi, D. B.; Feild, C. A.; Harrison, W. T. A.; Guloy, A. M. Conducting Tin Halides with a Layered Organic-Based Perovskite Structure. Nature 1994, 369 (6480), 467-469. https://doi.org/10.1038/369467a0.

(50) Sheldrick, G. M. SHELXT - Integrated Space-Group and Crystal-Structure Determination. Acta Crystallogr. Sect. A Found. Adv. 2015, 71 (1), 3-8. https://doi.org/10.1107/S2053273314026370.

(51) Sheldrick, G. M. Crystal Structure Refinement with SHELXL. Acta Crystallogr. Sect. C Struct. Chem. 2015, 71 (1), 3-8. https://doi.org/10.1107/S2053229614024218.

Dolomanov, O. V; Bourhis, L. J.; Gildea, R. J.; Howard, J. A. K.; Puschmann, H. 
OLEX2: A Complete Structure Solution, Refinement and Analysis Program. J. Appl. Crystallogr. 2009, 42 (2), 339-341. https://doi.org/10.1107/S0021889808042726.

(53) Momma, K.; Izumi, F. VESTA 3 for Three-Dimensional Visualization of Crystal, Volumetric and Morphology Data. J. Appl. Crystallogr. 2011, 44 (6), 1272-1276. https://doi.org/10.1107/S0021889811038970.

(54) Mitzi, D. B. Templating and Structural Engineering in Organic-inorganic Perovskites. J. Chem. Soc. Dalt. Trans. 2001, No. 1, 1-12. https://doi.org/10.1039/b007070j.

(55) Li, Q.; Zhou, Q.; Shi, L.; Chen, Q.; Wang, J. Recent Advances in Oxidation and Degradation Mechanisms of Ultrathin 2D Materials under Ambient Conditions and Their Passivation Strategies. J. Mater. Chem. A 2019, 7 (9), 4291-4312. https://doi.org/10.1039/C8TA10306B.

(56) Shi, E.; Deng, S.; Yuan, B.; Gao, Y.; Akriti; Yuan, L.; Davis, C. S.; Zemlyanov, D.; Yu, Y.; Huang, L.; et al. Extrinsic and Dynamic Edge States of Two-Dimensional Lead Halide Perovskites. ACS Nano 2019, acsnano.8b07631. https://doi.org/10.1021/acsnano.8b07631.

(57) Lee, G.-H.; Cui, X.; Kim, Y. D.; Arefe, G.; Zhang, X.; Lee, C.-H.; Ye, F.; Watanabe, K.; Taniguchi, T.; Kim, P.; et al. Highly Stable, Dual-Gated $\mathrm{MoS}_{2}$ Transistors Encapsulated by Hexagonal Boron Nitride with Gate-Controllable Contact, Resistance, and Threshold Voltage. ACS Nano 2015, 9 (7), 7019-7026. https://doi.org/10.1021/acsnano.5b01341.

(58) Ahn, S.; Kim, G.; Nayak, P. K.; Yoon, S. I.; Lim, H.; Shin, H.-J.; Shin, H. S. Prevention of Transition Metal Dichalcogenide Photodegradation by Encapsulation with h-BN Layers. ACS Nano 2016, 10 (9), 8973-8979. https://doi.org/10.1021/acsnano.6b05042.

(59) Kim, K. K.; Lee, H. S.; Lee, Y. H. Synthesis of Hexagonal Boron Nitride Heterostructures for 2D van Der Waals Electronics. Chem. Soc. Rev. 2018, 47 (16), 6342-6369. https://doi.org/10.1039/C8CS00450A.

(60) Amat, A.; Mosconi, E.; Ronca, E.; Quarti, C.; Umari, P.; Nazeeruddin, M. K.; Grätzel, M.; De Angelis, F. Cation-Induced Band-Gap Tuning in Organohalide Perovskites: Interplay of Spin-Orbit Coupling and Octahedra Tilting. Nano Lett. 2014, 14 (6), 3608-3616. https://doi.org/10.1021/nl5012992.

(61) Mao, L.; Guo, P.; Kepenekian, M.; Hadar, I.; Katan, C.; Even, J.; Schaller, R. D.; Stoumpos, C. C.; Kanatzidis, M. G. Structural Diversity in White-Light-Emitting Hybrid Lead Bromide Perovskites. J. Am. Chem. Soc. 2018, 140 (40), 13078-13088. https://doi.org/10.1021/jacs.8b08691.

(62) Mao, L.; Wu, Y.; Stoumpos, C. C.; Traore, B.; Katan, C.; Even, J.; Wasielewski, M. R.; Kanatzidis, M. G. Tunable White-Light Emission in Single-Cation-Templated ThreeLayered 2D Perovskites $\left(\mathrm{CH}_{3} \mathrm{CH}_{2} \mathrm{NH}_{3}\right)_{4} \mathrm{~Pb}_{3} \mathrm{Br}_{10-} \mathrm{Cl}_{x}$. J. Am. Chem. Soc. 2017, 139 (34), 11956-11963. https://doi.org/10.1021/jacs.7b06143.

(63) Alonso, J. A.; Martínez-Lope, M. J.; Casais, M. T.; Fernández-Díaz, M. T. Evolution of 
the Jahn-Teller Distortion of $\mathrm{MnO} 6$ Octahedra in $\mathrm{RMnO}_{3}$ Perovskites $(\mathrm{R}=\mathrm{Pr}, \mathrm{Nd}, \mathrm{Dy}$, Tb, Ho, Er, Y): A Neutron Diffraction Study. Inorg. Chem. 2000, 39 (5), 917-923. https://doi.org/10.1021/ic990921e.

(64) Lufaso, M. W.; Woodward, P. M. Jahn-Teller Distortions, Cation Ordering and Octahedral Tilting in Perovskites. Acta Crystallogr. Sect. B Struct. Sci. 2004, 60 (1), 1020. https://doi.org/10.1107/S0108768103026661.

(65) Robinson, K.; Gibbs, G. V.; Ribbe, P. H. Quadratic Elongation: A Quantitative Measure of Distortion in Coordination Polyhedra. Science. 1971, 172 (3983), 567-570. https://doi.org/10.1126/science.172.3983.567.

(66) Coe, B. J.; Glenwright, S. J. Trans-Effects in Octahedral Transition Metal Complexes. Coord. Chem. Rev. 2000, 203 (1), 5-80. https://doi.org/10.1016/S0010-8545(99)00184-8.

(67) Stoumpos, C. C.; Malliakas, C. D.; Kanatzidis, M. G. Semiconducting Tin and Lead Iodide Perovskites with Organic Cations: Phase Transitions, High Mobilities, and NearInfrared Photoluminescent Properties. Inorg. Chem. 2013, 52 (15), 9019-9038. https://doi.org/10.1021/ic401215x.

(68) Smith, M. D.; Pedesseau, L.; Kepenekian, M.; Smith, I. C.; Katan, C.; Even, J.; Karunadasa, H. I. Decreasing the Electronic Confinement in Layered Perovskites through Intercalation. Chem. Sci. 2017, 8 (3), 1960-1968. https://doi.org/10.1039/C6SC02848A. 
Table of Contents

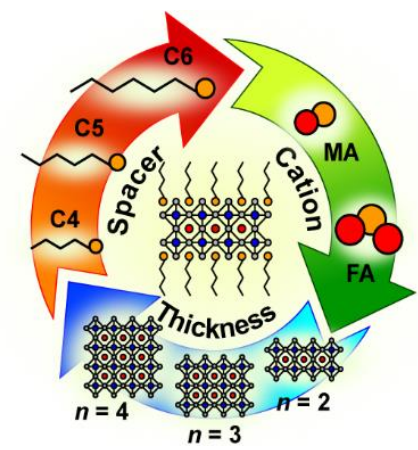


Supplementary Information for:

\title{
Synthetic Variation and Structural Trends in Layered Two- Dimensional Alkylammonium Lead Halide Perovskites
}

\author{
Watcharaphol Paritmongkol ${ }^{1,2}$, Nabeel S. Dahod ${ }^{2}$, Nannan $\mathrm{Mao}^{2,3}$, \\ Shao-Liang Zheng ${ }^{4}$, William A. Tisdale ${ }^{2 *}$ \\ ${ }^{1}$ Department of Chemistry, Massachusetts Institute of Technology, Cambridge, Massachusetts \\ 02139, United States \\ ${ }^{2}$ Department of Chemical Engineering, Massachusetts Institute of Technology, Cambridge, \\ Massachusetts 02139, United States \\ ${ }^{3}$ Department of Electrical Engineering and Computer Science, Massachusetts Institute of \\ Technology, Cambridge, Massachusetts 02139, United States \\ ${ }^{4}$ Department of Chemistry and Chemical Biology, Harvard University, Cambridge, Massachusetts \\ 02138, United States \\ *Correspondence to: tisdale@ mit.edu
}

\section{Contents}

Section S1. Synthesis conditions

Section S2. Synthesis observations

Section S3. Bright-field micrographs of exfoliated flakes

Section S4. Optical characterization

Section S5. Structural illustrations 


\section{Section S1. Synthesis conditions}

Table S1: Reagent quantities used for iodide 2D LHP syntheses.

\begin{tabular}{|c|c|c|c|c|c|c|c|}
\hline & $\begin{array}{c}n=1 \\
\text { BA-PbI }\end{array}$ & $\begin{array}{c}n=2 \\
\text { BA-MAPbI }\end{array}$ & $\begin{array}{c}n=3 \\
\text { BA-MAPbI }\end{array}$ & $\begin{array}{c}n=4 \\
\text { BA-MAPbI }\end{array}$ & $\begin{array}{c}n=2 \\
\text { PA-MAPbI }\end{array}$ & $\begin{array}{c}n=2 \\
\text { HA-MAPbI }\end{array}$ & $\begin{array}{c}n=2 \\
\text { BA-FAPbI }\end{array}$ \\
\hline PbO mass (g) & 0.558 & 0.558 & 0.558 & 0.558 & 0.558 & 0.279 & 0.558 \\
\hline $\begin{array}{l}\text { Number of moles } \\
\text { of PbO (mmol) }\end{array}$ & 2.5 & 2.5 & 2.5 & 2.5 & 2.5 & 1.25 & 2.5 \\
\hline $\begin{array}{l}\text { HI volume to } \\
\text { make } \mathrm{PbI}_{2} \text { solution } \\
(\mathrm{mL})\end{array}$ & 3 & 3 & 3 & 3 & 3 & 1.5 & 3 \\
\hline $\begin{array}{l}\mathrm{H}_{3} \mathrm{PO}_{2} \text { volume } \\
(\mu \mathrm{L})\end{array}$ & 425 & 425 & 425 & 425 & 425 & 213 & 425 \\
\hline AI mass (g) & - & 0.1987 & 0.2650 & 0.2981 & 0.1987 & 0.1739 & 0.2150 \\
\hline $\begin{array}{l}\text { Number of moles } \\
\text { of AI (mmol) }\end{array}$ & - & 1.25 & 1.67 & 1.875 & 1.25 & 1.09 & 1.25 \\
\hline $\begin{array}{l}\text { AI\% w.r.t. } \\
\text { stoichiometric } \\
\text { amount }\end{array}$ & - & 100 & 100 & 100 & 100 & 175 & 100 \\
\hline $\begin{array}{l}\text { HI volume to } \\
\text { make AI solution } \\
(\mathrm{mL})\end{array}$ & - & 0.75 & 0.75 & 0.75 & 0.75 & 1 & 0.75 \\
\hline L volume $(\boldsymbol{\mu L})$ & 247 & 161 & 107 & 49 & 174 & 83 & 161 \\
\hline $\begin{array}{l}\text { Number of moles } \\
\text { of L (mmol) }\end{array}$ & 2.5 & 1.625 & 0.997 & 0.5 & 1.5 & 0.625 & 1.625 \\
\hline $\begin{array}{l}\text { L\% w.r.t. } \\
\text { stoichiometric } \\
\text { amount }\end{array}$ & 50 & 65 & 65 & 40 & 60 & 50 & 65 \\
\hline $\begin{array}{l}\text { Additional volume } \\
\text { of } \mathrm{HI} \text { added to the } \\
\text { combined solution }\end{array}$ & 5 & 0 & 0 & 0 & 0.25 & 0 & 0.75 \\
\hline $\begin{array}{l}\text { Total volume of } \mathrm{HI} \\
\text { used }(\mathrm{mL})\end{array}$ & 8 & 3.75 & 3.75 & 3.75 & 4 & 2.5 & 4.5 \\
\hline
\end{tabular}


Table S2: Reagent quantities used for bromide 2D LHP syntheses.

\begin{tabular}{|c|c|c|c|}
\hline & $\begin{array}{c}n=1 \\
\text { BA-PbBr }\end{array}$ & $\begin{array}{c}n=2 \\
\text { BA- } \\
\text { MAPbBr }\end{array}$ & $\begin{array}{c}n=3 \\
\text { BA- } \\
\text { MAPbBr }\end{array}$ \\
\hline PbO mass (g) & 0.558 & 0.279 & 0.279 \\
\hline Number of moles of $\mathrm{PbO}(\mathrm{mmol})$ & 2.5 & 1.25 & 1.25 \\
\hline HBr volume to make $\mathrm{PbBr}_{2}$ solution $(\mathrm{mL})$ & 3 & 1.5 & 1.5 \\
\hline $\mathrm{ABr}$ mass $(\mathrm{g})$ & - & 0.0700 & 0.0933 \\
\hline Number of moles of $\mathrm{ABr}(\mathrm{mmol})$ & - & 0.625 & 0.833 \\
\hline ABr\% w.r.t. stoichiometric amount & - & 100 & 100 \\
\hline HBr volume to make ABr solution (mL) & - & 0.4 & 0.4 \\
\hline L volume $(\mu \mathrm{L})$ & 247 & 74 & 37 \\
\hline Number of moles of $\mathrm{L}(\mathrm{mmol})$ & 2.5 & 0.75 & 0.375 \\
\hline L\% w.r.t. stoichiometric amount & 50 & 60 & 45 \\
\hline $\begin{array}{l}\text { Additional volume of } \mathrm{HBr} \text { added to the } \\
\text { combined solution }\end{array}$ & 5 & 0.5 & 0.5 \\
\hline Total volume of $\mathrm{HBr}$ used $(\mathrm{mL})$ & 8 & 2.4 & 2.4 \\
\hline
\end{tabular}




\section{Section S2. Synthesis observations}

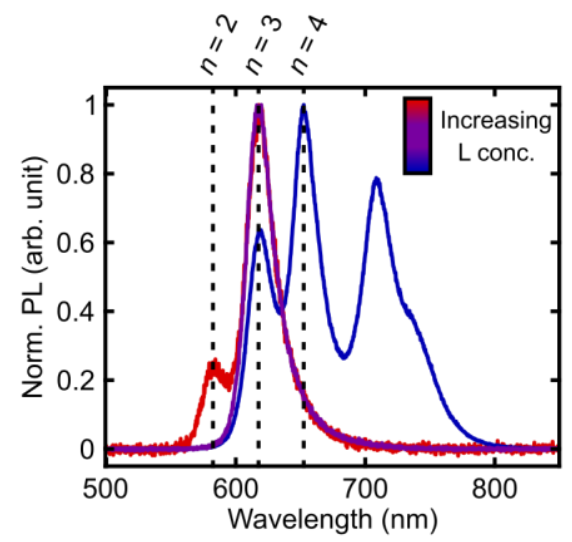

Figure S1. Photoluminescence spectra showing the effect of organic spacer (L) concentration. In this experiment, we attempted to synthesize pure $n=3 \mathrm{BA}-\mathrm{MAPbI}$. If the L concentration is too low, photoluminescence peaks due to $n=4 \mathrm{BA}-\mathrm{MAPbI}$ and higher members appear. On the other hand, if the L concentration is too high, photoluminescence peak due to $n=2 \mathrm{BA}-\mathrm{MAPbI}$ is observed.

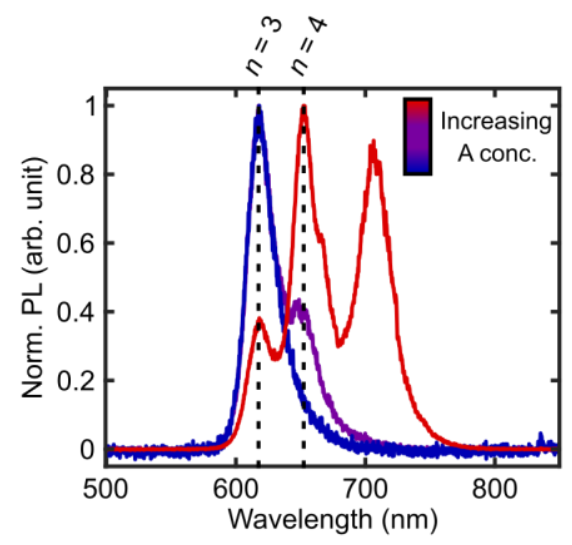

Figure S2. Photoluminescence spectra showing the effect of A-site cation concentration. In this experiment, we attempted to synthesize pure $n=3 \mathrm{BA}-\mathrm{MAPbI}$. If the A-site cation concentration is too high, photoluminescence peaks due to $n=4 \mathrm{BA}-\mathrm{MAPbI}$ and higher members appear. 


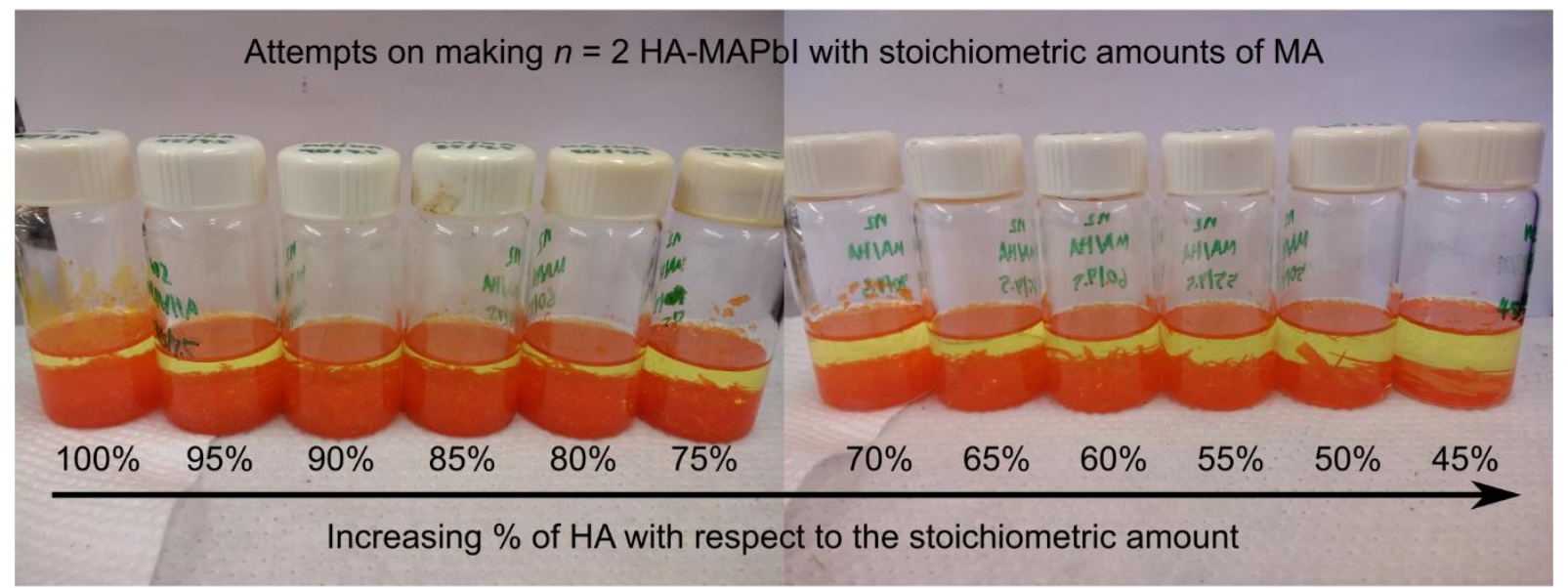

Figure S3. Crystals of $n=1 \mathrm{HA}-\mathrm{PbI}$ obtained as the main products from the syntheses of $n=2$ HA-MAPbI with $100 \%$ of MA stoichiometric amounts. Regardless of the amounts of HA used, only the orange crystals of $n=1 \mathrm{HA}-\mathrm{PbI}$ were yielded.

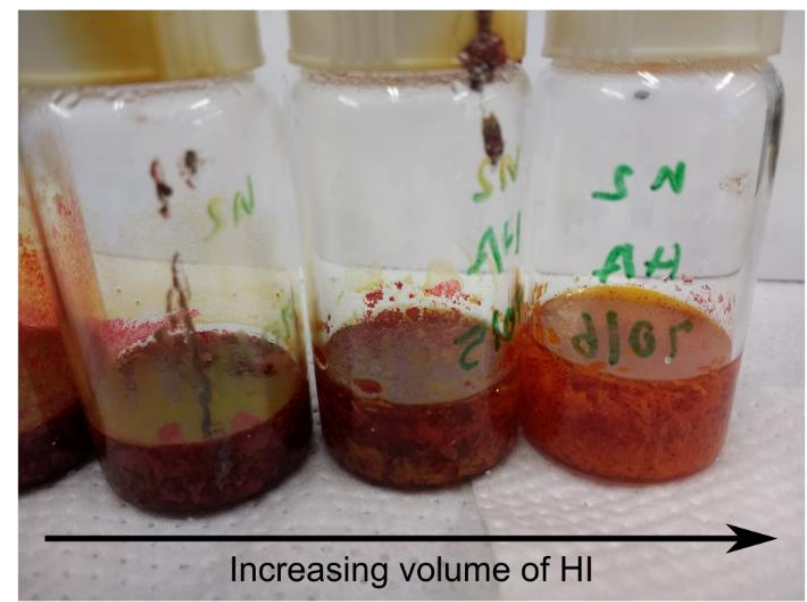

Figure S4. Picture of crystals in solutions showing the effect of HI volume to the synthesis products. When a small volume of HI is used, a crowded solution of $n=2 \mathrm{HA}-\mathrm{MAPbI}$ (cherry red crystals) is obtained. As the volume of HI increases, the solution is less crowded but the main products become orange crystal of $n=1 \mathrm{HA}-\mathrm{PbI}$. 
(a)

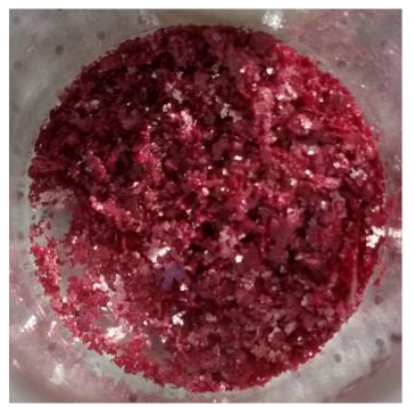

(b)

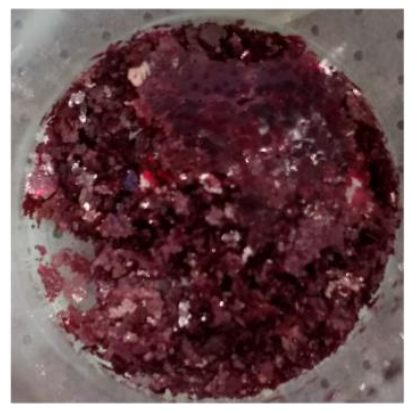

(c)

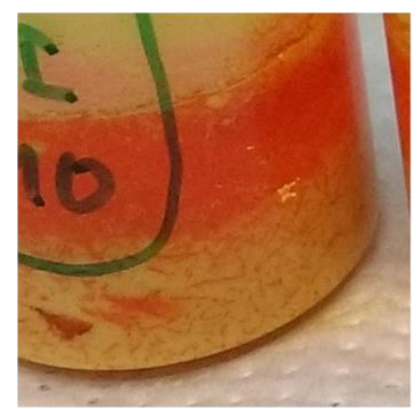

Figure S5. (a-b) Ruby-red crystals of $n=2 \mathrm{BA}-\mathrm{FAPbI}$ formed as the main products of the attempted syntheses of $n=3$ BA-FAPbI (a) and $n=4$ BA-FAPbI (b). (c) White solid of CsI and orange crystals of $n=1 \mathrm{BA}-\mathrm{PbI}$ were yielded from the attempted synthesis of $n=2 \mathrm{BA}-\mathrm{CsPbI}$.

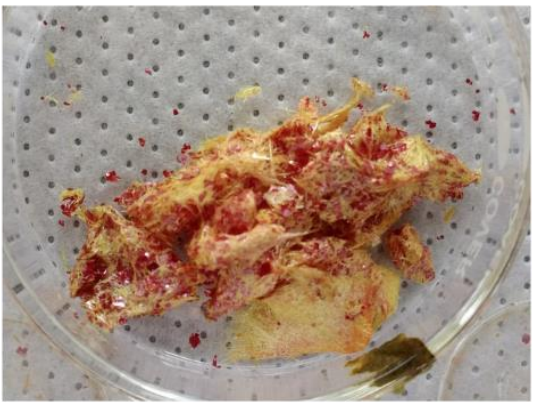

Figure S6. Picture of $n=2$ BA-MAPbI crystals with yellow, needle-like crystal by-products. 

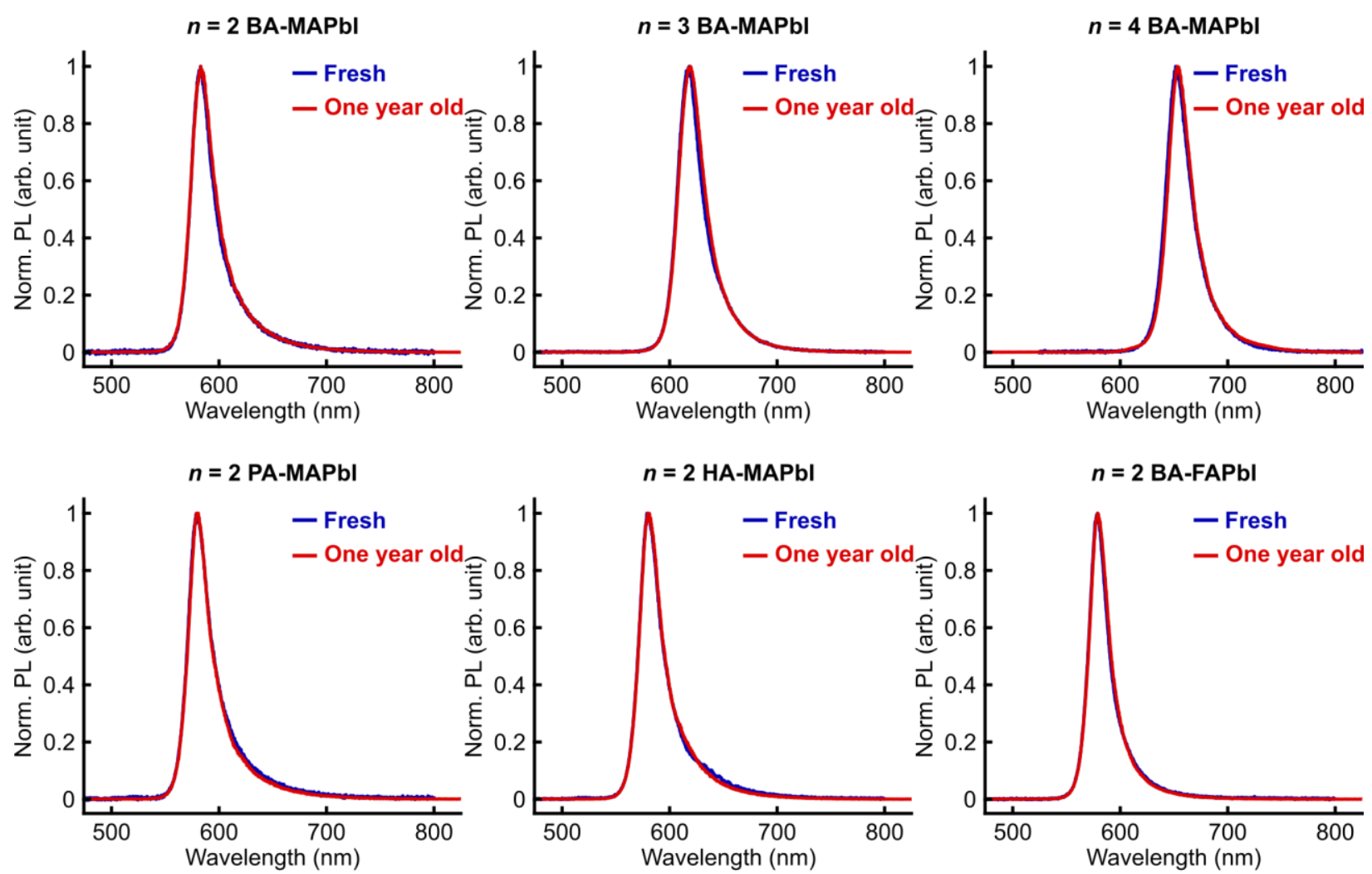

Figure S7. Effect of storage in $\mathrm{N}_{2}$ glovebox: photoluminescence spectra of fresh and one-year-old samples of $n=2$ BA-MAPbI, $n=3$ BA-MAPbI, $n=4$ BA-MAPbI, $n=2$ PA-MAPbI, $n=2$ HA$\mathrm{MAPbI}$, and $n=2 \mathrm{BA}-\mathrm{FAPbI}$. The one-year-old samples were stored in a $\mathrm{N}_{2}$ glovebox with $<10$ ppm $\mathrm{O}_{2}$ and $<1 \mathrm{ppm}_{2} \mathrm{O}$.

(a)

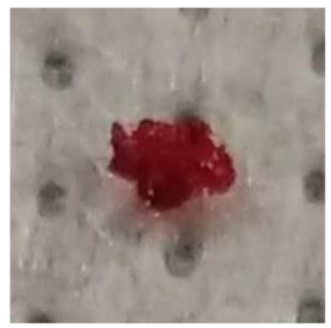

(b)

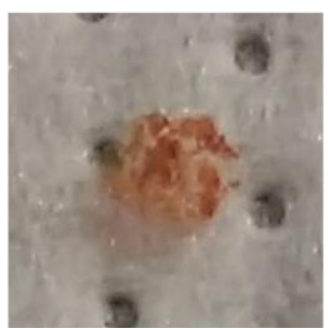

Figure S8. (a) Newly exfoliated flake of $n=2$ HA-MAPbI. (b) The same exfoliated flake after storing under ambient conditions for 3 days. 


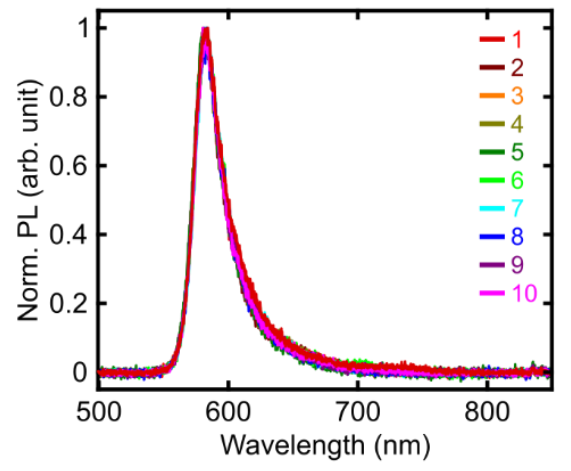

Figure S9. Photoluminescence spectra obtained at different locations on a high-purity sample of $n=2 \mathrm{BA}-\mathrm{MAPbI}$. All spectra show single emission peaks without impurity emission from iodide 2D LHPs with other quantum-well thicknesses.

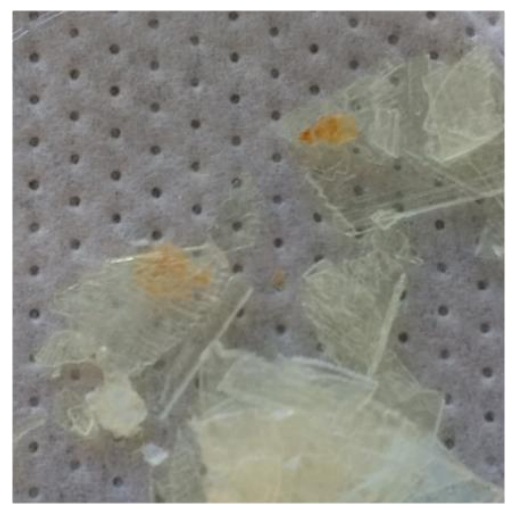

Figure S10. Orange coloring on white crystals of $n=1 \mathrm{BA}-\mathrm{PbBr}$. Because the synthesis of $n=1$ $\mathrm{BA}-\mathrm{PbBr}$ does not involve MA cation, these orange colorings are not believed to be due to the formation of bulk $\mathrm{MAPbBr}_{3}$ or higher members of bromide 2D LHPs. 
Section S3. Bright-field micrographs of exfoliated flakes

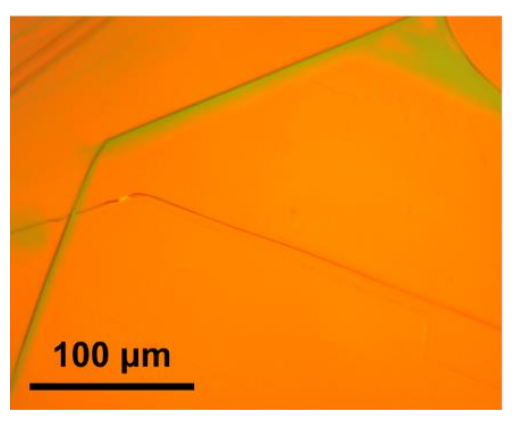

$n=2$ BA-MAPbI

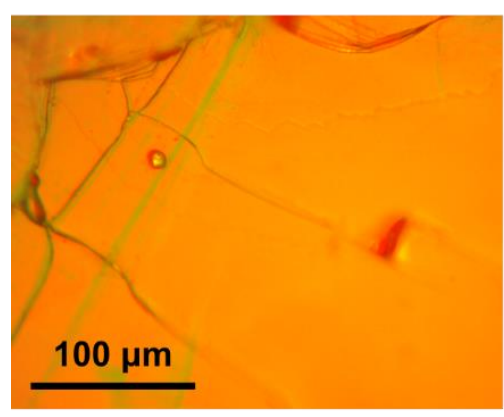

$n=2$ PA-MAPbI

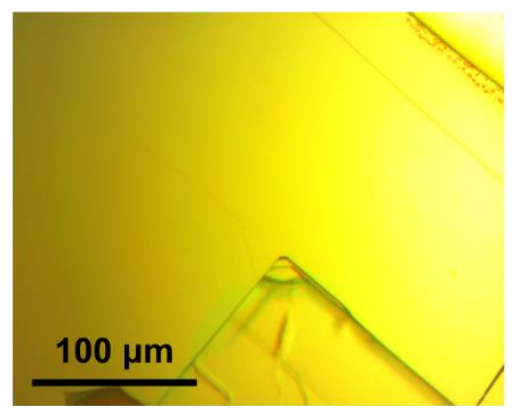

$n=3$ BA-MAPbl

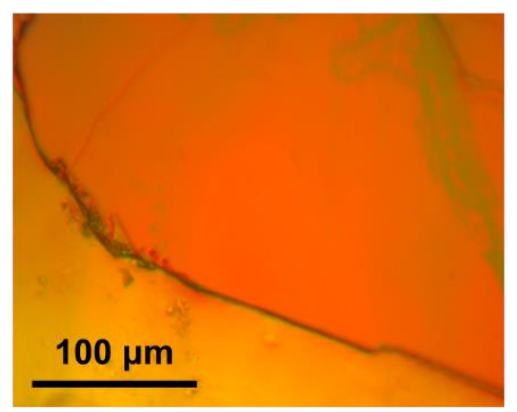

$n=2$ HA-MAPbl

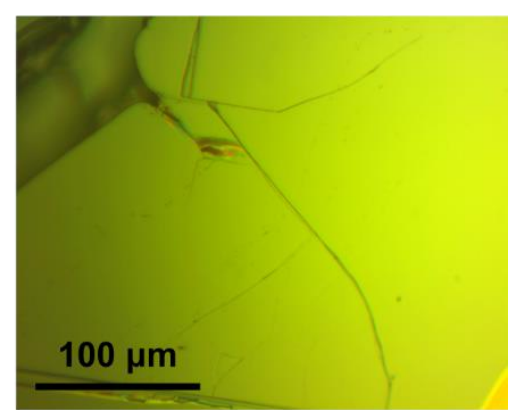

$n=4$ BA-MAPbI

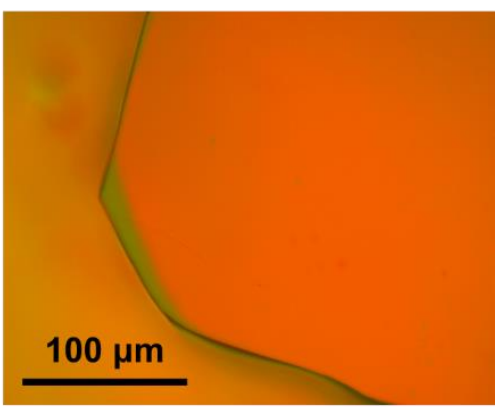

$n=2$ BA-FAPbl

Figure S11. Bright-filed micrographs of exfoliated flakes of 2D LHPs. 


\section{Section S4. Optical characterization}

Table S3. Summary of absorption (Abs) peak wavelengths, photoluminescence (PL) peak wavelengths, PL full width at half maxima (FWHM), and Stokes shifts of iodide and bromide 2D LHP crystals.

\begin{tabular}{|l|c|c|c|c|}
\hline \multicolumn{1}{|c|}{ Sample } & $\begin{array}{c}\text { Abs peak } \\
\text { wavelength } \\
(\mathrm{nm})\end{array}$ & $\begin{array}{c}\text { PL peak } \\
\text { wavelength } \\
(\mathrm{nm})\end{array}$ & $\begin{array}{c}\text { PL FWHM } \\
(\mathrm{nm})\end{array}$ & $\begin{array}{c}\text { Stokes shift } \\
(\mathrm{nm})\end{array}$ \\
\hline$n=1$ BA-PbI & $508 \pm 1$ & $522 \pm 1$ & $17 \pm 2$ & $14 \pm 2$ \\
\hline$n=2$ BA-MAPbI & $571 \pm 1$ & $582 \pm 1$ & $25 \pm 2$ & $11 \pm 2$ \\
\hline$n=3$ BA-MAPbI & $607 \pm 1$ & $617 \pm 1$ & $27 \pm 2$ & $10 \pm 2$ \\
\hline$n=4$ BA-MAPbI & $643 \pm 1$ & $652 \pm 1$ & $27 \pm 2$ & $9 \pm 2$ \\
\hline$n=2$ PA-MAPbI & $569 \pm 1$ & $579 \pm 1$ & $25 \pm 2$ & $10 \pm 2$ \\
\hline$n=2$ HA-MAPbI & $570 \pm 1$ & $580 \pm 1$ & $25 \pm 2$ & $10 \pm 2$ \\
\hline$n=2$ BA-FAPbI & $570 \pm 1$ & $578 \pm 1$ & $21 \pm 2$ & $8 \pm 2$ \\
\hline & & & & $17 \pm 2$ \\
\hline$n=1$ BA-PbBr & $406 \pm 1$ & $418 \pm 1$ & $17 \pm 2$ & $9 \pm 2$ \\
\hline$n=2$ BA-MAPbBr & $436 \pm 1$ & $445 \pm 1$ & $16 \pm 2$ & $10 \pm 2$ \\
\hline$n=3$ BA-MAPbBr & $456 \pm 1$ & $466 \pm 1$ & &
\end{tabular}




\section{Section S5. Structural illustrations}

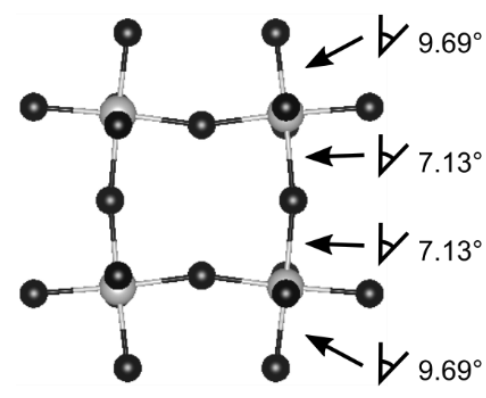

$n=2$ BA-MAPbI

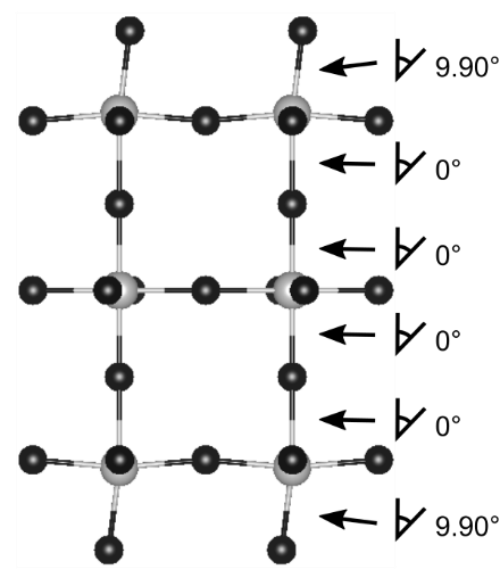

$n=3$ BA-MAPbI

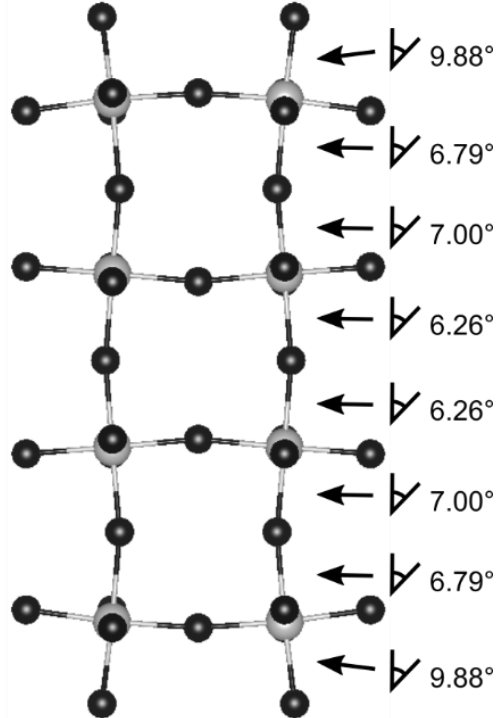

$n=4$ BA-MAPbI

Figure S12. Illustrations of $n=2 \mathrm{BA}-\mathrm{MAPbI}, n=3 \mathrm{BA}-\mathrm{MAPbI}$, and $n=4 \mathrm{BA}-\mathrm{MAPbI}$ showing out-of-plane tilting angles of axial $\mathrm{Pb}$-I bonds. The angels are measured with respect to the stacking direction. 


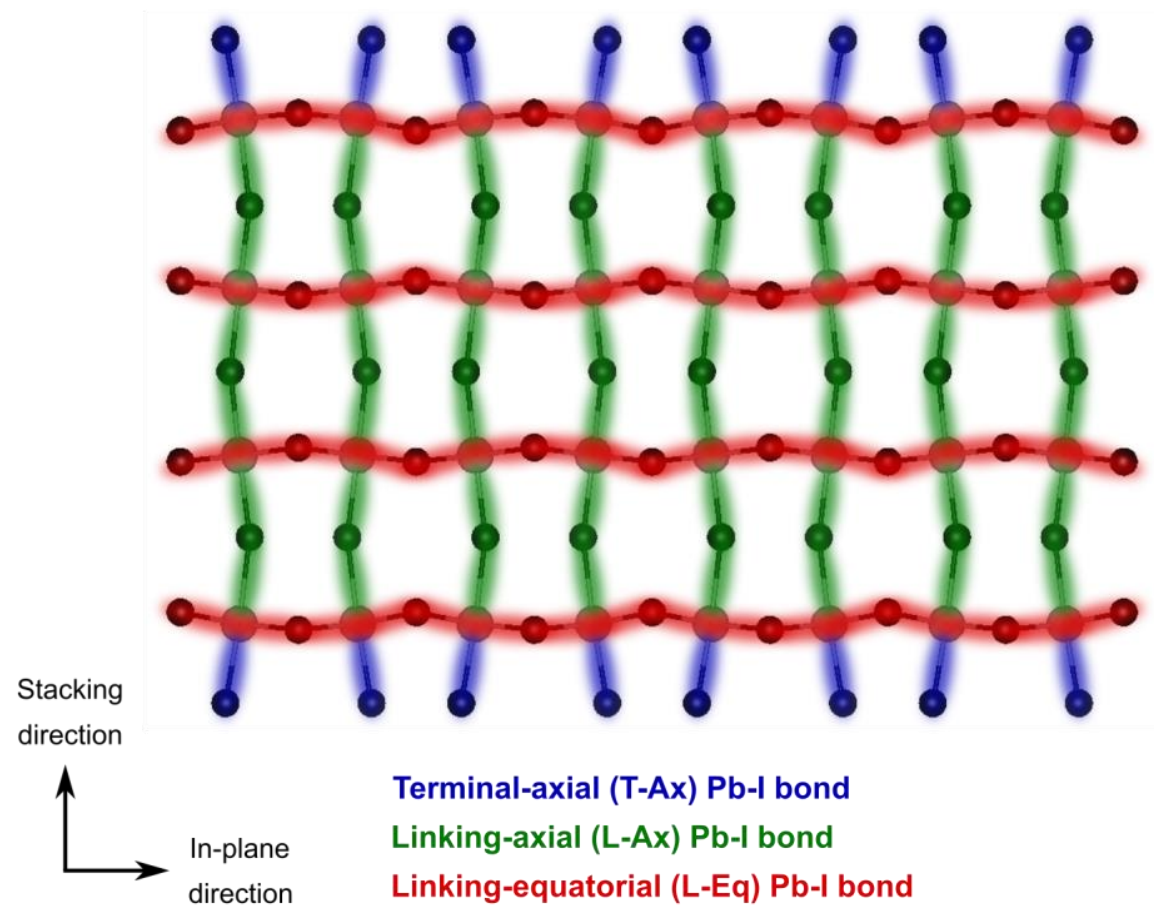

Figure S13. Illustration of an inorganic slab highlighting the three types of Pb-I bonds: terminalaxial (T-Ax), linking-axial (L-Ax), and linking-equatorial (L-Eq) bonds. 